\title{
SOBOLEV SPACES WITH RESPECT TO A WEIGHTED GAUSSIAN MEASURES IN INFINITE DIMENSIONS
}

\author{
S. FERRARI
}

\begin{abstract}
Let $X$ be a separable Banach space endowed with a non-degenerate centered Gaussian measure $\mu$ and let $w$ be a positive function on $X$ such that $w \in W^{1, s}(X, \mu)$ and $\log w \in W^{1, t}(X, \mu)$ for some $s>1$ and $t>s^{\prime}$. In the present paper we introduce and study Sobolev spaces with respect to the weighted Gaussian measure $\nu:=w \mu$. We obtain results regarding the divergence operator (i.e. the adjoint in $\mathrm{L}^{2}$ of the gradient operator along the Cameron-Martin space) and the trace of Sobolev functions on hypersurfaces $\{x \in X \mid G(x)=0\}$, where $G$ is a suitable version of a Sobolev function.
\end{abstract}

\section{INTRODUCTION}

Let $X$ be a separable Banach space with norm $\|\cdot\|_{X}$, endowed with a non-degenerate centered Gaussian measure $\mu$. The associated Cameron-Martin space is denoted by $H$, its inner product by $\langle\cdot, \cdot\rangle_{H}$ and its norm by $|\cdot|_{H}$. The spaces $W^{1, p}(X, \mu)$ and $W^{2, p}(X, \mu)$ for $p \geq 1$ are the classical Sobolev spaces of the Malliavin calculus (see [7]). The following hypothesis will be assumed throughout the paper:

Hypothesis 1.1. We will denote the weight function by $w$ and assume that $w(x)>0, \mu$-a.e. and

(1) $w \in W^{1, s}(X, \mu)$ for some $s>1$ and it is a $(1, s)$-precise version (see section 2);

(2) $\log w \in W^{1, t}(X, \mu)$ for some $t>s^{\prime}$, where $s^{\prime}$ denotes the conjugate exponent of $s$ (this technical hypothesis will be explained later).

We set

$$
\nu:=w \mu \text {. }
$$

We recall that $\nu$ is a Radon measure absolutely continuous with respect to $\mu$. The aim of this paper is to study the Sobolev spaces with respect to the measure $\nu$. In particular we study the divergence operator, i.e. the adjoint in $\mathrm{L}^{2}$ of the gradient operator, and the traces on hypersurfaces $\{x \in X \mid G(x)=0\}$ where $G: X \rightarrow \mathbb{R}$ is a suitable version of a Sobolev function. The main results about Sobolev spaces with respect to the Gaussian measure can be found in [7], while results about surface measures and traces of Sobolev functions in Gaussian Sobolev spaces can be found in [17, 16] and in [10], respectively. We will assume the following hypotheses about the "regularity" of the hypersurfaces we work with:

Hypothesis 1.2. Let $G \in W^{2, q}(X, \mu)$ be a $(2, q)$-precise version (see Section 2) for every $q>1$ and assume

(1) $\mu\left(G^{-1}(-\infty, 0)\right)>0$;

(2) there exists $\delta>0$ such that $\left|\nabla_{H} G\right|_{H}^{-1} \in \mathrm{L}^{q}\left(G^{-1}(-\delta, \delta), \mu\right)$ for every $q>1$.

Hypothesis 1.2(2) is classical, indeed see [2], [7] and [10], but it is difficult to prove. In this paper we are able to check Hypothesis 1.2(2) only for some notable functions $G$ (see Section 7).

One of our main results is an infinite dimensional weighted version of the divergence theorem, namely:

Date: September 20, 2017.

2010 Mathematics Subject Classification. 28C20, 46G12.

Key words and phrases. Infinite dimensional analysis, Traces, Weighted Gaussian measure, divergence operator, Weighted Sobolev spaces, Sublevel sets. 
Theorem 1.3. Let $p \geq \frac{t}{t-s^{\prime}}$ and $\left\{e_{k} \mid k \in \mathbb{N}\right\}$ be an orthonormal basis of $H$. For every $\varphi \in$ $W^{1, p}\left(G^{-1}(-\infty, 0), \nu\right)$ and $k \in \mathbb{N}$ we have

$$
\int_{G^{-1}(-\infty, 0)}\left(\partial_{k} \varphi+\varphi \partial_{k} \log w-\varphi \widehat{e}_{k}\right) d \nu=\int_{G^{-1}(0)} \operatorname{Tr}_{G^{-1}(0)}\left(\varphi \partial_{k} G\right) \frac{w}{\left|\nabla_{H} G\right|_{H}} d \rho
$$

Furthermore if $\Phi \in W^{1, p}\left(G^{-1}(-\infty, 0), \nu ; H\right)$ then

$$
\int_{G^{-1}(-\infty, 0)} \operatorname{div}_{\nu} \Phi d \nu=\int_{G^{-1}(0)}\left\langle\operatorname{Tr}_{G^{-1}(0)} \Phi, \operatorname{Tr}_{G^{-1}(0)} \nabla_{H} G\right\rangle_{H} \frac{w}{\left|\nabla_{H} G\right|_{H}} d \rho,
$$

where $\operatorname{Tr}_{G^{-1}(0)} \Psi=\sum_{n=1}^{+\infty}\left(\operatorname{Tr}_{G^{-1}(0)} \psi_{n}\right) e_{n}$ if $\Psi \in W^{1, p}\left(G^{-1}(-\infty, 0), \nu ; H\right)$ and $\psi_{n}=\left\langle\Psi, e_{n}\right\rangle$.

The functions $\left\{\widehat{e}_{k} \mid k \in \mathbb{N}\right\}$ and the partial derivative $\partial_{k}$ will be defined in Section 2, the spaces $W^{1, p}\left(G^{-1}(-\infty, 0), \nu\right)$ and $W^{1, p}\left(G^{-1}(-\infty, 0), \nu ; H\right)$ will be defined in section 5 , the trace operator $\operatorname{Tr}_{G^{-1}(0)}$ will be introduced in section 6 and the divergence operator $\operatorname{div}_{\nu}$ will be studied in Section 4 .

Weighted Gaussian measures are an important part of infinite dimensional analysis. They arise in many problems in stochastic analysis (see for example [13] and [11]) and in obstacle problems theory (see for example [20]). We used the theory of Sobolev spaces with respect to a log-concave weighted Gaussian measure in order to prove maximal Sobolev regularity estimates (see [8] and [9]) and to characterize the domain of elliptic operators in Wiener spaces (see [1]).

The paper is organized in the following way: in Section 2 we will introduce the basic notations that we will use throughout the paper.

In Section 3 we will introduce the Sobolev spaces $W^{1, p}(X, \nu)$ and study their basic properties. We will show that the following integration by parts formula holds:

$$
\int_{X} \partial_{h} f(x) d \nu(x)=\int_{X} f(x)\left(\widehat{h}(x)-\partial_{h} \log w(x)\right) d \nu(x) \quad \text { for every } f \in \mathscr{F} \mathscr{C}_{b}^{\infty}(X) \text { and } h \in H,
$$

where $\mathscr{F} \mathscr{C}_{b}^{\infty}(X)$ denotes the vector space of the smooth cylindrical functions (the functions of the form $f(x)=\varphi\left(l_{1}(x), \ldots, l_{n}(x)\right)$, for some $\varphi \in \mathscr{C}_{b}^{\infty}\left(\mathbb{R}^{n}\right), l_{1}, \ldots, l_{n} \in X^{*}$ and $\left.n \in \mathbb{N}\right)$, while $\widehat{h}$ and $\partial_{h}$ will be defined in 2. Some of the results of this section can be found in [2], where a more general class of weights were consider on the space $\mathscr{C}[0,1]$.

In Section 4 we will introduce the divergence operator $\operatorname{div}_{\nu}$ as minus the formal adjoint of the gradient operator along $H$ and investigate some of its basic properties. In Proposition 4.3 we will prove that under suitable hypotheses on the weight (namely (4.4)) then $W^{1,2}(X, \nu ; H)$ is contained in the domain of $\operatorname{div}_{\nu}$ and $\operatorname{div}_{\nu} \Phi \in \mathrm{L}^{2}(X, \nu)$ for every $\Phi \in W^{1,2}(X, \nu ; H)$. Example 4.4 shows that the conditions in Proposition 4.3 are only sufficient to ensure that the domain of $\operatorname{div}_{\nu}$ is not empty. Finally Proposition 4.5 shows that if $\Phi \in W^{1, q}(X, \mu ; H)$ (the Gaussian Sobolev spaces) then $\operatorname{div}_{\nu} \Phi$ belongs to $\mathrm{L}^{p}(X, \nu)$ for some reasonable values of $p$. Furthermore an explicit formula for the calculation of $\operatorname{div}_{\nu}$ is given in (4.8).

In Section 5 we will introduce the Sobolev spaces on sets $G^{-1}(-\infty, 0)$ in a similar way as in Section 3 , where $G$ is a function satisfying Hypothesis 1.2.

In Section 6 we will introduce the trace $\operatorname{Tr}_{G^{-1}(0)} f$ of a Sobolev function $f$ in $W^{1, p}\left(G^{-1}(-\infty, 0), \nu\right)$ on $G^{-1}(0)$, where $G$ is a function satisfying Hypothesis 1.2. In this section we give the proof of our main result (Theorem 1.3) and we show in Proposition 6.5, that if $\bar{\varphi}$ is a $(1, p)$-precise version (see Section 2) of an element $\varphi$ in $W^{1, p}\left(G^{-1}(-\infty, 0), \nu\right)$, then

$$
\operatorname{Tr}_{G^{-1}(0)} \varphi=\bar{\varphi}_{\left.\right|_{G^{-1}(0)}} \quad \rho \text {-a.e. }
$$

where $\rho$ is the Feyel-de La Pradelle Hausdorff-Gauss surface measure introduced in [16] (see also Section 5 for more informations).

In Section 7 we will show how our results can be applied to explicit examples. The chosen hypersurface will be the unit sphere and the hyperplanes. In Example 7.1 we study the weights $w_{\lambda}(x)=$ $\exp \left(\lambda\|x\|_{X}^{2}\right)$, where $X$ is a separable Hilbert space and $\lambda$ is a real number. In example 7.2 we study 
the weights $w_{q}(x)=\exp \left(\|x\|_{q}\right)$ in $\ell_{2}$, where $q>1$ and

$$
\|x\|_{q}=\left(\sum_{i=1}^{+\infty}|x(i)|^{q}\right)^{\frac{1}{q}}
$$

We show that every $w_{q}$ satisfies Hypothesis 1.1 (note that $\|\cdot\|_{q}$ is not continuous if $1<q<2$ ). Eventually in Example 7.3 we study the weight $w(f)=\exp \left(\|f\|_{\infty}\right)$ in the space $\mathscr{C}[0,1]$. In this case we will consider two type of surfaces: the hyperplanes and $\left\{f \in \mathscr{C}[0,1] \mid\|f\|_{2}=1\right\}$. All the examples are concluded by some observations about the continuity of the trace operator from $W^{1, p}\left(G^{-1}(-\infty, 0), \nu\right)$ to $\mathrm{L}^{q}\left(G^{-1}(0), w \rho\right)$ for $q \in[1, p]$, where $\rho$ is the Feyel-de La Pradelle Hausdorff-Gauss surface measure.

\section{Notation AND PRELIMINARIES}

We will denote by $X^{*}$ the topological dual of $X$. We recall that $X^{*} \subseteq \mathrm{L}^{2}(X, \mu)$. The linear operator $R_{\mu}: X^{*} \rightarrow\left(X^{*}\right)^{\prime}$

$$
R_{\mu} x^{*}\left(y^{*}\right)=\int_{X} x^{*}(x) y^{*}(x) d \mu(x)
$$

is called the covariance operator of $\mu$. Since $X$ is separable, then it is actually possible to prove that $R_{\mu}: X^{*} \rightarrow X$ (see [7, Theorem 3.2.3]). We denote by $X_{\mu}^{*}$ the closure of $X^{*}$ in $\mathrm{L}^{2}(X, \mu)$. The covariance operator $R_{\mu}$ can be extended by continuity to the space $X_{\mu}^{*}$, still by (2.1). By [7, Lemma 2.4.1] for every $h \in H$ there exists a unique $g \in X_{\mu}^{*}$ with $h=R_{\mu} g$, in this case we set

$$
\widehat{h}:=g
$$

Throughout the paper we fix an orthonormal basis $\left\{e_{i}\right\}_{i \in \mathbb{N}}$ of $H$ such that $\widehat{e}_{i}$ belongs to $X^{*}$, for every $i \in \mathbb{N}$. Such basis exists by [7, Corollary 3.2.8(ii)].

2.1. Differentiability along $H$. We say that a function $f: X \rightarrow \mathbb{R}$ is differentiable along $H$ at $x$ if there exists $v \in H$ such that

$$
\lim _{t \rightarrow 0} \frac{f(x+t h)-f(x)}{t}=\langle v, h\rangle_{H},
$$

uniformly with respect to $h \in H$, with $|h|_{H}=1$. In this case, the vector $v \in H$ is unique and we set $\nabla_{H} f(x):=v$. Moreover, for every $k \in \mathbb{N}$ the derivative of $f$ in the direction of $e_{k}$ exists and it is given by

$$
\partial_{k} f(x):=\lim _{t \rightarrow 0} \frac{f\left(x+t e_{k}\right)-f(x)}{t}=\left\langle\nabla_{H} f(x), e_{k}\right\rangle_{H}
$$

We denote by $\mathcal{H}_{2}$ the space of the Hilbert-Schmidt operators in $H$, that is the space of the bounded linear operators $A: H \rightarrow H$ such that $\|A\|_{\mathcal{H}_{2}}^{2}=\sum_{i}\left|A e_{i}\right|_{H}^{2}$ is finite (see [15]). We say that a function $f: X \rightarrow \mathbb{R}$ is twice differentiable along $H$ at $x$ if it is differentiable along $H$ at $x$ and there exists $A \in \mathcal{H}_{2}$ such that

$$
H-\lim _{t \rightarrow 0} \frac{\nabla_{H} f(x+t h)-\nabla_{H} f(x)}{t}=A h,
$$

uniformly with respect to $h \in H$, with $|h|_{H}=1$. In this case the operator $A$ is unique and we set $\nabla_{H}^{2} f(x):=A$. Moreover, for every $i, j \in \mathbb{N}$ we set

$$
\partial_{i j} f(x):=\lim _{t \rightarrow 0} \frac{\partial_{j} f\left(x+t e_{i}\right)-\partial_{j} f(x)}{t}=\left\langle\nabla_{H}^{2} f(x) e_{j}, e_{i}\right\rangle_{H}
$$


2.2. Special classes of functions. For $k \in \mathbb{N} \cup\{\infty\}$, we denote by $\mathscr{F} \mathscr{C}^{k}(X)\left(\mathscr{F} \mathscr{C}_{b}^{k}(X)\right.$ respectively) the space of the cylindrical function of the type $f(x)=\varphi\left(x_{1}^{*}(x), \ldots, x_{n}^{*}(x)\right)$ where $\varphi \in \mathscr{C}^{k}\left(\mathbb{R}^{n}\right)$ $\left(\varphi \in \mathscr{C}_{b}^{k}\left(\mathbb{R}^{n}\right)\right.$, respectively) and $x_{1}^{*}, \ldots, x_{n}^{*} \in X^{*}$, for some $n \in \mathbb{N}$. We remark that by a classical argument it is possible to prove that $\mathscr{F}_{\mathscr{C}} \mathscr{C}_{b}^{\infty}(X)$ is dense in $\mathrm{L}^{p}(X, \nu)$ for all $p \geq 1$ (see [12]). We recall that if $f \in \mathscr{F} \mathscr{C}^{2}(X)$, then $\partial_{i j} f(x)=\partial_{j i} f(x)$ for every $i, j \in \mathbb{N}$ and $x \in X$.

Let $L_{p}$ be the infinitesimal generator of the Ornstein-Uhlenbeck semigroup $\mathcal{T}(t)$ in $\mathrm{L}^{p}(X, \mu)$, where

$$
T(t) f(x):=\int_{X} f\left(e^{-t} x+\left(1-e^{-2 t}\right)^{\frac{1}{2}} y\right) d \mu(y) \quad \text { for } t>0 .
$$

For $k=1,2,3$, we define the $C_{k, p}$-capacity of an open set $A \subseteq X$ as

$$
C_{k, p}(A):=\inf \left\{\|f\|_{\mathrm{L}^{p}(X, \mu)} \mid\left(I-L_{p}\right)^{-\frac{k}{2}} f \geq 1 \mu \text {-a.e. in } A\right\} .
$$

For a general Borel set $B \subseteq X$ we let $C_{k, p}(B)=\inf \left\{C_{k, p}(A) \mid B \subseteq A\right.$ open $\}$. By $f \in W^{k, p}(X, \mu)$ we mean an equivalence class of functions and we call every element "version". For any $f \in W^{k, p}(X, \mu)$ there exists a version $\bar{f}$ of $f$ which is Borel measurable and $C_{k, p}$-quasicontinuous, i.e. for every $\varepsilon>0$ there exists an open set $A \subseteq X$ such that $C_{k, p}(A) \leq \varepsilon$ and $\bar{f}_{\mid X \backslash A}$ is continuous. Furthermore, for every $r>0$

$$
C_{k, p}(\{x \in X|| \bar{f}(x) \mid>r\}) \leq \frac{1}{r}\left\|\left(I-L_{p}\right)^{-\frac{k}{2}} \bar{f}\right\|_{\mathrm{L}^{p}(X, \mu)} .
$$

See [7, Theorem 5.9.6]. Such a version is called a $(k, p)$-precise version of $f$. Two precise versions of the same $f$ coincide outside sets with null $C_{k, p}$-capacity. All our results will be independent on our choice of a precise version of $G$ in Hypothesis 1.2. With obvious modification the same definition can be adapted to functions belonging to $W^{k, p}(X, \mu ; H)$ and $W^{k, p}\left(X, \mu ; \mathcal{H}_{2}\right)$.

2.3. Sobolev spaces. The Gaussian Sobolev spaces $W^{1, p}(X, \mu)$ and $W^{2, p}(X, \mu)$, with $p \geq 1$, are the completions of the smooth cylindrical functions $\mathscr{F} \mathscr{C}_{b}^{\infty}(X)$ in the norms

$$
\begin{gathered}
\|f\|_{W^{1, p}(X, \mu)}:=\|f\|_{L^{p}(X, \mu)}+\left(\int_{X}\left|\nabla_{H} f(x)\right|_{H}^{p} d \mu(x)\right)^{\frac{1}{p}} \\
\|f\|_{W^{2, p}(X, \mu)}:=\|f\|_{W^{1, p}(X, \mu)}+\left(\int_{X}\left\|\nabla_{H}^{2} f(x)\right\|_{\mathcal{H}_{2}}^{p} d \mu(x)\right)^{\frac{1}{p}} .
\end{gathered}
$$

Such spaces can be identified with subspaces of $\mathrm{L}^{p}(X, \mu)$ and the (generalized) gradient and Hessian along $H, \nabla_{H} f$ and $\nabla_{H}^{2} f$, are well defined and belong to $\mathrm{L}^{p}(X, \mu ; H)$ and $\mathrm{L}^{p}\left(X, \mu ; \mathcal{H}_{2}\right)$, respectively. The spaces $W^{1, p}(X, \mu ; H)$ are defined in a similar way, replacing smooth cylindrical functions with $H$-valued smooth cylindrical functions (i.e. the linear span of the functions $x \mapsto f(x) h$, where $f$ is a smooth cylindrical function and $h \in H$ ). For more information see [7, Section 5.2].

We remind the reader that the following integration by parts formula holds for every $f \in W^{1, p}(X, \mu)$, with $p>1$, and $h \in H$

$$
\int_{X} \partial_{h} f(x) d \mu(x)=\int_{X} f(x) \widehat{h}(x) d \mu(x) .
$$

2.4. Surface measures. For a comprehensive treatment of surface measures in infinite dimensional Banach spaces with Gaussian measures we refer to [17], [16] and [10]. We recall the definition of the Feyel-de La Pradelle Hausdorff-Gauss surface measure. If $m \geq 2$ and $F=\mathbb{R}^{m}$ equipped with a norm $\|\cdot\|_{F}$, we define

$$
d \theta^{F}(x)=\frac{1}{(2 \pi)^{\frac{m}{2}}} e^{-\frac{\|x\|_{F}^{2}}{2}} d H_{m-1}(x),
$$

where $H_{m-1}$ is the spherical $(m-1)$-dimensional Hausdorff measure in $\mathbb{R}^{m}$, i.e.

$$
H_{m-1}(A)=\lim _{\delta \rightarrow 0} \inf \left\{\sum_{n \in \mathbb{N}} w_{m-1} r_{n}^{m-1} \mid A \subseteq \bigcup_{n \in \mathbb{N}} B\left(x_{n}, r_{n}\right), r_{n}<\delta, \text { for every } n \in \mathbb{N}\right\},
$$


where $w_{m-1}=\pi^{\frac{m-1}{2}}\left(\Gamma\left(\frac{m+1}{2}\right)\right)^{-1}$. For every $m$-dimensional $F \subseteq H$ we consider the orthogonal projection (along $H$ ) on $F$ :

$$
x \mapsto \sum_{n=1}^{m}\left\langle x, f_{n}\right\rangle_{H} f_{n} \quad x \in H
$$

where $\left\{f_{n}\right\}_{n=1}^{m}$ is an orthonormal basis of $F$. There exists a $\mu$-measurable projection $\pi^{F}$ on $F$, defined in the whole $X$, that extends it (see [7, Theorem 2.10.11]). We denote by $\widetilde{F}:=\operatorname{ker} \pi^{F}$ and by $\mu_{\widetilde{F}}$ the image of the measure $\mu$ on $\widetilde{F}$ through $I-\pi^{F}$. Finally we denote by $\mu_{F}$ the image of the measure $\mu$ on $F$ through $\pi^{F}$, which is the standard Gaussian measure on $\mathbb{R}^{m}$ if we identify $F$ with $\mathbb{R}^{m}$. Let $A \subseteq X$ be a Borel set and identify $F$ with $\mathbb{R}^{m}$, we set

$$
\rho^{F}(A):=\int_{\operatorname{ker} \pi^{F}} \theta^{F}\left(A_{x}\right) d \mu_{\widetilde{F}}(x),
$$

where $A_{x}=\{y \in F \mid x+y \in A\}$. The map $F \mapsto \rho^{F}(A)$ is well defined and increasing, namely if $F_{1} \subseteq F_{2}$ are finite dimensional subspaces of $H$, then $\rho^{F_{1}}(A) \leq \rho^{F_{2}}(A)$ (see [3, Lemma 3.1] and [16, Proposition 3.2]). The Feyel-de La Pradelle Hausdorff-Gauss surface measure is defined by

$$
\rho(A)=\sup \left\{\rho^{F}(A) \mid F \subseteq H, F \text { is a finite dimensional subspace }\right\} .
$$

\section{Weighted Sobolev SPACES}

We want to define the Sobolev space $W^{1, p}(X, \nu)$ as the domain of the closure of the gradient operator along $H$. A natural procedure is to prove an integration by parts formula.

Lemma 3.1. Let $f \in \mathscr{F}_{b}^{\infty}(X)$ and $h \in H$. The following formula holds:

$$
\int_{X} \partial_{h} f(x) d \nu(x)=\int_{X} f(x)\left(\widehat{h}(x)-\partial_{h} \log w(x)\right) d \nu(x) .
$$

Proof. Using the integration by parts formula for the Gaussian measure (2.2) we get

$$
\begin{aligned}
& \int_{X} \partial_{h} f(x) d \nu(x)=\int_{X} \partial_{h} f(x) w(x) d \mu(x)=\int_{X} \partial_{h}(f(x) w(x)) d \mu(x)-\int_{X} f(x) \partial_{h} w(x) d \mu(x)= \\
& =\int_{X} \widehat{h}(x) f(x) w(x) d \mu(x)-\int_{X} f(x) \frac{\partial_{h} w(x)}{w(x)} w(x) d \mu(x)=\int_{X} f(x)\left(\widehat{h}(x)-\partial_{h} \log w(x)\right) d \nu(x) .
\end{aligned}
$$

We are now ready to prove the closability of the gradient operator along $H$.

Proposition 3.2. The operator $\nabla_{H}: \mathscr{F} \mathscr{C}_{b}^{\infty}(X) \rightarrow L^{p}(X, \nu ; H)$ is closable in $L^{p}(X, \nu)$, whenever $p \geq \frac{t}{t-s^{\prime}}$.

Proof. Let $\left(f_{k}\right)_{k \in \mathbb{N}} \subseteq \mathscr{F} \mathscr{C}_{b}^{\infty}(X)$ be such that

$$
\begin{aligned}
& \lim _{k \rightarrow+\infty} f_{k}=0 \quad \text { in } L^{p}(X, \nu) ; \\
& \lim _{k \rightarrow+\infty} \nabla_{H} f_{k}=\Phi \quad \text { in } L^{p}(X, \nu ; H) .
\end{aligned}
$$

We want to prove that $\Phi(x)=0, \nu$-a.e. To this aim we will show that

$$
\int_{X}\left\langle\Phi(x), e_{n}\right\rangle_{H} u(x) d \nu(x)=0
$$

for every $n \in \mathbb{N}$ and $u \in \mathscr{F} \mathscr{C}_{b}^{\infty}(X)$. Recall that if $f, g \in \mathscr{F} \mathscr{C}_{b}^{\infty}(X)$, then $f g \in \mathscr{F} \mathscr{C}_{b}^{\infty}(X)$. By the integration by parts formula (Lemma 3.1), we get

$$
\int_{X} \partial_{n} f_{k}(x) u(x) d \nu(x)=\int_{X} f_{k}(x)\left(\widehat{e}_{n}(x)-\partial_{n} \log w(x)\right) u(x) d \nu(x)-\int_{X} f_{k}(x) \partial_{n} u(x) d \nu(x) .
$$


By the Hölder inequality we get

$$
\begin{gathered}
\int_{X}\left|\partial_{n} f_{k}(x)-\left\langle\Phi(x), e_{n}\right\rangle\right||u(x)| d \nu(x) \leq \\
\leq\left(\int_{X}\left|\partial_{n} f_{k}(x)-\left\langle\Phi(x), e_{n}\right\rangle\right|^{p} d \nu(x)\right)^{\frac{1}{p}}\left(\int_{X}|u(x)|^{p^{\prime}} d \nu(x)\right)^{\frac{1}{p^{\prime}}} \stackrel{k \rightarrow+\infty}{\longrightarrow} 0 .
\end{gathered}
$$

Furthermore

$$
\int_{X}\left|f_{k}(x) \partial_{n} u(x)\right| d \nu(x) \leq\left(\int_{X}\left|f_{k}(x)\right|^{p} d \nu(x)\right)^{\frac{1}{p}}\left(\int_{X}\left|\partial_{n} u(x)\right|^{p^{\prime}} d \nu(x)\right)^{\frac{1}{p^{\prime}} \stackrel{k \rightarrow+\infty}{\longrightarrow} 0 .}
$$

Lastly

$$
\begin{gathered}
\int_{X}\left|f_{k}\right|\left|\widehat{e}_{n}-\partial_{n} \log w\left\|u\left|d \nu \leq\|u\|_{\infty} \int_{X}\right| f_{k}|| \widehat{e}_{n}-\partial_{n} \log w \mid d \nu \leq{ }_{(1)}\right.\right. \\
\leq{ }_{(1)}\|u\|_{\infty}\left(\int_{X}\left|f_{k}\right|^{p} d \nu\right)^{\frac{1}{p}}\left(\int_{X}\left|\widehat{e}_{n}-\partial_{n} \log w\right|^{p^{\prime}} w d \mu\right)^{\frac{1}{p^{\prime}}} \leq{ }_{(2)} \\
\leq_{(2)}\|u\|_{\infty}\left(\int_{X}\left|f_{k}\right|^{p} d \nu\right)^{\frac{1}{p}}\left(\int_{X} w^{s} d \mu\right)^{\frac{1}{s}}\left(\int_{X}\left|\widehat{e}_{n}-\partial_{n} \log w\right|^{p^{\prime} s^{\prime}} d \mu\right)^{\frac{1}{p^{\prime} s^{\prime}}} \stackrel{k \rightarrow+\infty}{\longrightarrow} 0
\end{gathered}
$$

where both (1) and (2) follow applying the Hölder inequality. Note that the last integral is finite whenever $p^{\prime} s^{\prime} \leq t$, i.e. $p \geq \frac{t}{t-s^{\prime}}$ which is an assumption. Letting $k \rightarrow+\infty$ in (3.2) we get

$$
\int_{X}\left\langle\Phi(x), e_{n}\right\rangle u(x) d \nu(x)=0
$$

for every $n \in \mathbb{N}$ and $u \in \mathscr{F} \mathscr{C}_{b}^{\infty}(X)$.

Definition 3.3 (Weighted Sobolev spaces). Let $p \geq \frac{t}{t-s^{\prime}}$. We denote by $W^{1, p}(X, \nu)$ the domain of the closure of $\nabla_{H}$ (which we still denote by the symbol $\nabla_{H}$ ) in $L^{p}(X, \nu)$. It is a Banach space with the graph norm

$$
\|f\|_{W^{1, p}(X, \nu)}=\left(\int_{X}|f(x)|^{p} d \nu(x)\right)^{\frac{1}{p}}+\left(\int_{X}\left|\nabla_{H} f(x)\right|_{H}^{p} d \nu(x)\right)^{\frac{1}{p}}
$$

In the same way we define $W^{1, p}(X, \nu ; H)$ using $H$-valued smooth cylindrical functions. Furthermore Lemma 3.1 holds for every $f \in W^{1, p}(X, \nu)$.

Using a standart argument it is possible to prove that (3.1) holds for every $f \in W^{1, p}(X, \nu)$, with $p \geq \frac{t}{t-s^{\prime}}$, and $h \in H$. The first thing we want to show is the following result about the coincidence of the closure of the gradient operator along $H$ in $\mathrm{L}^{p}(X, \nu)$ and $\mathrm{L}^{q}(X, \mu)$.

Proposition 3.4. In this proposition we will denote by $\nabla_{H}^{\mu, q}$ and $\nabla_{H}^{\nu, p}$ the closure of the gradient operator along $H$ in $\mathrm{L}^{q}(X, \mu)$ and $\mathrm{L}^{p}(X, \nu)$, respectively. Let $p \geq \frac{t}{t-s^{\prime}}$ and $\left(f_{n}\right)_{n \in \mathbb{N}} \subseteq \mathscr{F} \mathscr{C}_{b}^{\infty}(X)$. If there exists $f \in W^{1, q}(X, \mu)$, for some $q>p s^{\prime}$, such that

$$
\begin{gathered}
\lim _{n \rightarrow+\infty} f_{n}=f \quad \text { in } \mathrm{L}^{q}(X, \mu) ; \\
\lim _{n \rightarrow+\infty} \nabla_{H} f_{n}=\nabla_{H}^{\mu, q} f \quad \text { in } \mathrm{L}^{q}(X, \mu ; H),
\end{gathered}
$$

then

$$
\begin{gathered}
\lim _{n \rightarrow+\infty} f_{n}=f \quad \text { in } \mathrm{L}^{p}(X, \nu) ; \\
\lim _{n \rightarrow+\infty} \nabla_{H} f_{n}=\nabla_{H}^{\nu, p} f \quad \text { in } \mathrm{L}^{p}(X, \nu ; H) .
\end{gathered}
$$

Furthermore $\nabla_{H}^{\nu, p} f=\nabla_{H}^{\mu, q} f \mu$-a.e. 
Proof. By the Hölder inequality we get

$$
\begin{gathered}
\int_{X}\left|f_{n}-f\right|^{p} d \nu \leq\left(\int_{X} w^{s} d \mu\right)^{\frac{1}{s}}\left(\int_{X}\left|f_{n}-f\right|^{p s^{\prime}} d \mu\right)^{\frac{1}{s^{\prime}}} \leq \\
\leq\left(\int_{X} w^{s} d \mu\right)^{\frac{1}{s}}\left(\int_{X}\left|f_{n}-f\right|^{q} d \mu\right)^{\frac{p}{q}} \stackrel{n \rightarrow+\infty}{\longrightarrow} 0 .
\end{gathered}
$$

So $\lim _{n \rightarrow+\infty} f_{n}=f$ in $\mathrm{L}^{p}(X, \nu)$. In the same way

$$
\begin{gathered}
\int_{X}\left|\nabla_{H} f_{n}-\nabla_{H}^{\mu, q} f\right|_{H}^{p} d \nu \leq\left(\int_{X} w^{s} d \mu\right)^{\frac{1}{s}}\left(\int_{X}\left|\nabla_{H} f_{n}-\nabla_{H}^{\mu, q} f\right|_{H}^{p s^{\prime}} d \mu\right)^{\frac{1}{s^{\prime}}} \leq \\
\leq\left(\int_{X} w^{s} d \mu\right)^{\frac{1}{s}}\left(\int_{X}\left|\nabla_{H} f_{n}-\nabla_{H}^{\mu, q} f\right|_{H}^{q} d \mu\right)^{\frac{p}{q}} \stackrel{n \rightarrow+\infty}{\longrightarrow} 0 .
\end{gathered}
$$

So $\lim _{n \rightarrow+\infty} \nabla_{H} f_{n}=\nabla_{H}^{\mu, q} f$ in $\mathrm{L}^{p}(X, \nu ; H)$. The furthermore part is now obvious.

It is important to observe some basic properties of the space $W^{1, p}(X, \nu)$.

Proposition 3.5. Let $p \geq \frac{t}{t-s^{\prime}}$. The following holds:

(1) $W^{1, p}(X, \nu)$ is reflexive;

(2) for every $q \in\left(p s^{\prime},+\infty\right), W^{1, q}(X, \mu) \hookrightarrow W^{1, p}(X, \nu)$. In particular if $G$ satisfies Hypothesis 1.2 then $G \in W^{1, b}(X, \nu)$ for every $b \geq \frac{t}{t-s^{\prime}}$. The same is true for the spaces $W^{1, q}(X, \mu ; H)$ and $W^{1, p}(X, \nu ; H)$;

(3) if $\int_{X} w^{r(r-1)^{-1}} d \mu<+\infty$ for some $r \in(0,1)$, then $W^{1, p}(X, \nu) \hookrightarrow W^{1, p r}(X, \mu)$. The same is true for the space $W^{1, p}(X, \nu ; H)$;

(4) let $\left(\varphi_{n}\right)_{n \in \mathbb{N}} \subseteq W^{1, p}(X, \nu)$. If $\varphi_{n}$ converges pointwise $\nu$-a.e. to $\varphi$ and

$$
\sup _{n \in \mathbb{N}}\left\|\varphi_{n}\right\|_{W^{1, p}(X, \nu)}<+\infty
$$

then $\varphi \in W^{1, p}(X, \nu)$;

(5) let $q \geq \frac{t}{t-s^{\prime}}, \varphi \in W^{1, p}(X, \nu)$ and $\psi \in W^{1, q}(X, \nu)$. If $\frac{p q}{p+q} \geq \frac{t}{t-s^{\prime}}$ then

$$
\varphi \psi \in W^{1, r}(X, \nu) \quad \text { for every } r \in\left[\frac{t}{t-s^{\prime}}, \frac{p q}{p+q}\right] \text {, }
$$

and $\nabla_{H}(\varphi \psi)=\varphi \nabla_{H} \psi+\psi \nabla_{H} \varphi$.

Proof. (1) It is easily seen that $\mathrm{L}^{p}(X, \nu) \times \mathrm{L}^{p}(X, \nu ; H)$ is a reflexive Banach space when endowed with the norm

$$
\|(f, \Phi)\|=\|f\|_{\mathrm{L}^{p}(X, \nu)}+\|\Phi\|_{\mathrm{L}^{p}(X, \nu ; H)} .
$$

This is due to the fact that the weakly closed and norm bounded sets of both $\mathrm{L}^{p}(X, \nu)$ and $\mathrm{L}^{p}(X, \nu ; H)$ are weakly compact (see [15, Corollary IV.1.2]). Thus the set

$$
\left\{(f, \Phi) \in \mathrm{L}^{p}(X, \nu) \times \mathrm{L}^{p}(X, \nu ; H) \mid\|(f, \Phi)\| \leq 1\right\}
$$

is weakly compact and so $\mathrm{L}^{p}(X, \nu) \times \mathrm{L}^{p}(X, \nu ; H)$ is reflexive. The operator $T: W^{1, p}(X, \nu) \rightarrow$ $\mathrm{L}^{p}(X, \nu) \times \mathrm{L}^{p}(X, \nu ; H)$ defined as

$$
T(f)=\left(f, \nabla_{H} f\right),
$$

is an isometric embedding, which implies that the range of $T$ is closed in $\mathrm{L}^{p}(X, \nu) \times \mathrm{L}^{p}(X, \nu ; H)$. Thus $T\left(W^{1, p}(X, \nu)\right)$ is reflexive, being a closed subspace of a reflexive space. So $W^{1, p}(X, \nu)$ is reflexive too, being isometric to a reflexive space.

(2) Let $f \in W^{1, q}(X, \mu)$. Using the Hölder inequality we get

$$
\int_{X}|f|^{p} d \nu=\int_{X}|f|^{p} w d \mu \leq\left(\int_{X}|f|^{p s^{\prime}} d \mu\right)^{\frac{1}{s^{\prime}}}\left(\int_{X} w^{s} d \mu\right)^{\frac{1}{s}},
$$


and the right hand side is finite whenever $p s^{\prime}<q$. Using Proposition 3.4, the same inequality holds for $\nabla_{H} f$. Thus the statement follows.

(3) Let $f \in W^{1, p}(X, \nu)$. Using the Hölder inequality we get

$$
\int_{X}|f|^{p r} d \mu=\int_{X} \frac{|f|^{p r}}{w} d \nu \leq\left(\int_{X}|f|^{p} d \nu\right)^{r}\left(\int_{X} w^{\frac{r}{r-1}} d \mu\right)^{1-r} .
$$

Using the same argument in Proposition 3.4 it is possible to prove the same inequality for $\nabla_{H} f$. Thus the statement follows.

(4) The statement is a consequence of the Banach-Saks property, i.e. every bounded sequence $\left(\varphi_{n}\right)_{n \in \mathbb{N}} \subseteq \mathrm{L}^{p}(X, \nu ; H)$ has a subsequence $\left(\varphi_{n_{k}}\right)_{k \in \mathbb{N}}$ such that the sequence

$$
\left(\frac{\varphi_{n_{1}}+\cdots+\varphi_{n_{k}}}{k}\right)_{k \in \mathbb{N}}
$$

strongly converges in $\mathrm{L}^{p}(X, \nu ; H)$. The proof is the same of [7, Lemma 5.4.4] with obvious adjustments.

(5) Standard calculations.

Proposition 3.6. Let $p \geq \frac{t}{t-s^{\prime}}$ and $\theta \in \mathscr{C}_{b}^{1}(\mathbb{R})$. If $\varphi \in W^{1, p}(X, \nu)$, then $\theta \circ \varphi \in W^{1, p}(X, \nu)$ and

$$
\nabla_{H}(\theta \circ \varphi)=\left(\theta^{\prime} \circ \varphi\right) \nabla_{H} \varphi \quad \nu \text {-a.e. }
$$

Proof. Let $\left(\varphi_{n}\right)_{n \in \mathbb{N}} \subseteq \mathscr{F} \mathscr{C}_{b}^{\infty}(X)$ be such that $\varphi_{n}$ converges in $W^{1, p}(X, \nu)$ and pointwise $\nu$-a.e. to $\varphi$. Applying (4) of Proposition 3.5 to the sequence $\theta \circ \varphi_{n}$ we get $\theta \circ \varphi \in W^{1, p}(X, \nu)$. Furthermore we know that, for every $n \in \mathbb{N}, \theta \circ \varphi_{n} \in \mathscr{F} \mathscr{C}_{b}^{1}(X)$ and

$$
\nabla_{H}\left(\theta \circ \varphi_{n}\right)=\left(\theta^{\prime} \circ \varphi_{n}\right) \nabla_{H} \varphi_{n} .
$$

By Proposition 3.2 and the fact that $\theta \in \mathscr{C}_{b}^{1}(\mathbb{R})$, we can let $n \rightarrow+\infty$ and get the statement.

Proposition 3.7. Let $p \geq \frac{t}{t-s^{\prime}}$ and $u \in W^{1, p}(X, \nu)$. Then $|u| \in W^{1, p}(X, \nu)$ and

$$
\nabla_{H}|u|=\operatorname{sign}(u) \nabla_{H} u \text {. }
$$

Furthermore $\nabla_{H} u(x)=0$-a.e. in $\{y \in X \mid u(y)=0\}$.

Proof. The proof is the same as in [12, Lemma 2.7], we just use Lemma 3.1 instead of the classical integration by parts formula for the Gaussian measure (2.2).

\section{Divergence operator}

We start this section by recalling the definition of divergence, see [7, Section 5.8]. For every measurable map $\Phi: X \rightarrow X$ and for every $f \in \mathscr{F} \mathscr{C}_{b}^{\infty}(X)$ we define

$$
\partial_{\Phi} f(x)=\lim _{t \rightarrow 0} \frac{f(x+t \Phi(x))-f(x)}{t}, \quad x \in X .
$$

Definition 4.1. Let $\Phi \in \mathrm{L}^{1}(X, \nu ; X)$ be a vector field. We say that $\Phi$ admits divergence if there exists a function $g \in \mathrm{L}^{1}(X, \nu)$ such that

$$
\int_{X} \partial_{\Phi} f d \nu=-\int_{X} f g d \nu \quad \text { for every } f \in \mathscr{F} \mathscr{C}_{b}^{\infty}(X)
$$

where $\left(\partial_{\Phi} f\right)(x)$ is defined in (4.1). If such a function exists then we set

$$
\operatorname{div}_{\nu} \Phi:=g .
$$

Observe that, when $\operatorname{div}_{\nu} \Phi$ exists, it is unique by the density of $\mathscr{F}_{\mathscr{C}}^{\infty}(X)$. We denote by $D\left(\operatorname{div}_{\nu}\right)$ the domain of $\operatorname{div}_{\nu}$ in $\mathrm{L}^{1}(X, \nu ; X)$. Lastly observe that if $\Phi \in \mathrm{L}^{1}(X, \nu ; H)$, then $\left(\partial_{\Phi} f\right)(x)=\left\langle\nabla_{H} f(x), \Phi(x)\right\rangle_{H}$. In this case (4.2) becomes

$$
\int_{X}\left\langle\nabla_{H} f(x), \Phi(x)\right\rangle_{H} d \nu=-\int_{X} f g d \nu \quad \text { for every } f \in \mathscr{F} \mathscr{C}_{b}^{\infty}(X) .
$$


We are now interested in conditions ensuring the non emptyness of $D\left(\operatorname{div}_{\nu}\right)$ and $\mathrm{L}^{p}$-integrability of $\operatorname{div}_{\nu} v$. We start by studying the case of a vector field in $W^{1,2}(X, \nu ; H)$.

Proposition 4.2. Assume Hypothesis 1.1 holds and that $\log w \in W^{2, t}(X, \mu)$, where $t$ is the exponent fixed in Hypothesis 1.1. For every $f, g \in \mathscr{F} \mathscr{C}_{b}^{\infty}(X)$ and $h, k \in H$

$$
\begin{aligned}
& \int_{X}\left(f \widehat{h}-f \partial_{h} \log w-\partial_{h} f\right)\left(g \widehat{k}-g \partial_{k} \log w-\partial_{k} g\right) d \nu= \\
& =\langle h, k\rangle_{H} \int_{X} f g d \nu-\int_{X} f g \partial_{h} \partial_{k} \log w d \nu+\int_{X} \partial_{k} f \partial_{h} g d \nu .
\end{aligned}
$$

Proof. By the integration by parts formula (Lemma 3.1) we get

$$
\begin{aligned}
& \int_{X}\left(f \widehat{h}-f \partial_{h} \log w-\partial_{h} f\right)\left(g \widehat{k}-g \partial_{k} \log w-\partial_{k} g\right) d \nu= \\
& =\int_{X} f \partial_{h}(g \widehat{k}) d \nu-\int_{X} f \partial_{h}\left(g \partial_{k} \log w\right) d \nu-\int_{X} f \partial_{h} \partial_{k} g d \nu .
\end{aligned}
$$

Recalling that $\partial_{h} \widehat{k}=\langle h, k\rangle_{H}$ we get

$$
\begin{aligned}
& \int_{X}\left(f \widehat{h}-f \partial_{h} \log w-\partial_{h} f\right)\left(g \widehat{k}-g \partial_{k} \log w-\partial_{k} g\right) d \nu= \\
& =\langle h, k\rangle_{H} \int_{X} f g d \nu-\int_{X} f g \partial_{h} \partial_{k} \log w d \nu+\int_{X} \partial_{k} f \partial_{h} g d \nu .
\end{aligned}
$$

Proposition 4.3. Let $\log w \in W^{2, t}(X, \mu)$, for some $t \geq 2 s^{\prime}$, and assume that there exists $C \geq 0$ such that for every $\left(\xi_{i}\right)_{i \in \mathbb{N}} \in \ell_{2}$ we have

$$
\sum_{i=1}^{+\infty} \sum_{j=1}^{+\infty}\left(\delta_{i j}-\partial_{i} \partial_{j} \log w(x)\right) \xi_{i} \xi_{j} \leq C \sum_{i=1}^{+\infty} \xi_{i}^{2} \quad \text {-a.e. }
$$

Then every field $\Phi \in W^{1,2}(X, \nu ; H)$ has a divergence $\operatorname{div}_{\nu} \Phi \in L^{2}(X, \nu)$ and for every $f \in W^{1,2}(X, \nu)$, the following equality holds:

$$
\int_{X}\left\langle\nabla_{H} f(x), \Phi(x)\right\rangle_{H} d \nu(x)=-\int_{X} f(x) \operatorname{div}_{\nu} \Phi(x) d \nu(x) .
$$

Furthermore, if $\varphi_{n}=\left\langle\Phi, e_{n}\right\rangle_{H}$ for every $n \in \mathbb{N}$ where $\left(e_{n}\right)_{n \in \mathbb{N}}$ is an orthonormal basis of $H$, then

$$
\operatorname{div}_{\nu} \Phi(x)=\sum_{n=1}^{+\infty}\left(\partial_{n} \varphi_{n}(x)+\varphi_{n}(x) \partial_{n} \log w(x)-\varphi_{n}(x) \widehat{e}_{n}\right),
$$

and $\left\|\operatorname{div}_{\nu} \Phi\right\|_{\mathrm{L}^{2}(X, \nu)} \leq \max \{\sqrt{C}, 1\}\|\Phi\|_{W^{1,2}(X, \nu ; H)}$.

Proof. Let $\Phi(x)=\sum_{i=1}^{n} \varphi_{i}(x) e_{i}$ with $\varphi_{i} \in \mathscr{F} \mathscr{C}_{b}^{\infty}(X)$ for every $i=1, \ldots, n$. Then by the integration by parts formula (Lemma 3.1) if $f \in \mathscr{F} \mathscr{C}_{b}^{\infty}(X)$ we have

$$
\int_{X}\left\langle\nabla_{H} f(x), \Phi(x)\right\rangle_{H} d \nu(x)=-\int_{X} f(x)\left(\sum_{i=1}^{n}\left(\partial_{i} \varphi_{i}(x)+\varphi_{i}(x) \partial_{i} \log w(x)-\varphi_{i}(x) \widehat{e}_{i}\right)\right) d \nu(x) .
$$

Put

$$
\operatorname{div}_{\nu} \Phi(x)=\sum_{i=1}^{n}\left(\partial_{i} \varphi_{i}(x)+\varphi_{i}(x) \partial_{i} \log w(x)-\varphi_{i}(x) \widehat{e}_{i}\right)
$$


By Proposition 4.2

$$
\begin{gathered}
\int_{X}\left(\operatorname{div}_{\nu} \Phi\right)^{2} d \nu=\sum_{i=1}^{n} \int_{X}\left|\varphi_{i}\right|^{2} d \nu+\sum_{i=1}^{n} \sum_{j=1}^{n} \int_{X} \partial_{j} \varphi_{i} \partial_{i} \varphi_{j} d \nu-\sum_{i=1}^{n} \sum_{j=1}^{n} \int_{X} \varphi_{i} \varphi_{j} \partial_{i} \partial_{j} \log w d \nu= \\
=\int_{X} \sum_{i=1}^{n} \sum_{j=1}^{n}\left(\delta_{i j}-\partial_{i} \partial_{j} \log w\right) \varphi_{i} \varphi_{j} d \nu+\int_{X} \operatorname{trace}_{H}\left(\left(\nabla_{H} \Phi(x)\right)^{2}\right) d \nu(x) \leq \\
\leq C\|\Phi\|_{L^{2}(X, \nu ; H)}^{2}+\int_{X}\left\|\nabla_{H} \Phi(x)\right\|_{\mathcal{H}_{2}}^{2} d \nu(x) \leq \max \{C, 1\}\|\Phi\|_{W^{1,2}(X, \nu ; H)}^{2} .
\end{gathered}
$$

Let $\left(\Phi^{n}\right)_{n \in \mathbb{N}}$ be a sequence of fields as above which converges to $\Phi$ in $W^{1,2}(X, \nu ; H)$. The sequence $\left(\operatorname{div}_{\nu} \Phi^{n}\right)$ is Cauchy in $\mathrm{L}^{2}(X, \nu)$ and, hence it converges to some element, which is the candidate to be $\operatorname{div}_{\nu} \Phi$. By the integration by parts formula (Lemma 3.1) it is easily seen that such an element satifies (4.3). Finally, the fact that (4.3) holds also for every $f \in W^{1,2}(X, \nu)$, follows by a standard approximation argument.

Observe that (4.4) is satisfied whenever $\nabla_{H}^{2} \log w$ is bounded from below $\mu$-a.e., in particular when $\log w$ is a convex function.

The following example show that (4.4) is not necessary for the domain of the divergence to be not empty.

Example 4.4. Let $X$ be a separable Hilbert space, with norm $\|\cdot\|_{X}$ and inner product $(\cdot, \cdot)_{X}$, endowed with a nondegenerate centered Gaussian measure $\mu$, with covariance $Q$. We fix an orthonormal basis $\left(v_{n}\right)_{n \in \mathbb{N}}$ of $X$ of eigenvectors of $Q$, i.e. $Q v_{k}=\lambda_{k} v_{k}$, and the corresponding orthonormal basis of $H=Q^{\frac{1}{2}}(X)$ is $\left(e_{n}:=\sqrt{\lambda_{n}} v_{n}\right)_{n \in \mathbb{N}}$. Recall that $\widehat{e}_{n}(x)=\frac{\left(x, v_{n}\right)_{X}}{\sqrt{\lambda_{n}}}$. In this example we take $\lambda_{n}=4^{-n}$.

Let $w(x):=(x, x)_{X}^{2}=\sum_{n=1}^{+\infty}\left(x, v_{n}\right)_{X}^{2}=\sum_{n=1}^{+\infty} \lambda_{n}^{-1}\left(x, e_{n}\right)_{X}^{2}$. Easy calculations give

$$
\begin{gathered}
\partial_{i} w(x)=4(x, x)_{X}\left(x, e_{i}\right)_{X}, \quad \partial_{i} \log w(x)=4 \frac{\left(x, e_{i}\right)_{X}}{(x, x)_{X}}, \\
\partial_{i} \partial_{j} \log w(x)=4\left(\frac{\left(e_{i}, e_{j}\right)_{X}}{(x, x)_{X}}-\frac{2\left(x, e_{i}\right)_{X}\left(x, e_{j}\right)_{X}}{(x, x)_{X}^{2}}\right) .
\end{gathered}
$$

We get $w \in W^{1, s}(X, \mu)$ and $\log w \in W^{2, t}(X, \mu)$ for every $t, s>1$. Therefore $W^{1, p}(X, \nu)$ is well defined for every $p>1$ (see Definition 3.3).

We want to show that $w$ does not satisfy (4.4). For every $\left(\xi_{i}\right)_{i \in \mathbb{N}} \in \ell_{2}$ we have

$$
\begin{gathered}
\sum_{i=1}^{+\infty} \sum_{j=1}^{+\infty}\left(\delta_{i j}-\partial_{i} \partial_{j} \log w(x)\right) \xi_{i} \xi_{j}=\sum_{i=1}^{+\infty} \sum_{j=1}^{+\infty}\left(\delta_{i j}-4 \frac{\left(e_{i}, e_{j}\right)_{X}}{(x, x)_{X}}+8 \frac{\left(x, e_{i}\right)_{X}\left(x, e_{j}\right)_{X}}{(x, x)_{X}^{2}}\right) \xi_{i} \xi_{j}= \\
=\sum_{i=1}^{+\infty} \sum_{j=1}^{+\infty}\left(\delta_{i j}-4 \delta_{i j} \frac{\sqrt{\lambda_{i} \lambda_{j}}}{(x, x)_{X}}+8 \frac{\sqrt{\lambda_{i} \lambda_{j}}\left(x, v_{i}\right)_{X}\left(x, v_{j}\right)_{X}}{(x, x)_{X}^{2}}\right) \xi_{i} \xi_{j} .
\end{gathered}
$$

By contradiction assume that $C \geq 0$ exists such that (4.4) holds. Choosing $\xi_{i}=\left(x, v_{i}\right)$ for every fixed $x \in X$, we get

$$
\begin{gathered}
\sum_{i=1}^{+\infty} \sum_{j=1}^{+\infty}\left(\delta_{i j}-\partial_{i} \partial_{j} \log w(x)\right) \xi_{i} \xi_{j}= \\
=\sum_{i=1}^{+\infty}\left(x, v_{i}\right)_{X}^{2}-\frac{4}{(x, x)_{X}} \sum_{i=1}^{+\infty} \frac{\left(x, v_{i}\right)_{X}^{2}}{4^{i}}+\frac{8}{(x, x)_{X}^{2}}\left(\sum_{i=1}^{+\infty} \frac{\left(x, v_{i}\right)_{X}^{2}}{2^{i}}\right)^{2} \leq C \sum_{i=1}^{+\infty}\left(x, v_{i}\right)_{X}^{2} \quad \mu \text {-a.e.. }
\end{gathered}
$$

Let $A=\left\{x \in X \mid\|x\|_{X}^{2}<\sqrt{2^{-1}} \sum_{i=1}^{+\infty} 4^{-i}\left(x, v_{i}\right)_{X}^{2}\right\}$ and $B_{r}=\left\{x \in X \mid\|x\|_{X}<r\right\}$ for $0<r<1$. Observe that $A \cap B_{r}$ are open non-empty subsets of $X$ for every $r \in(0,1)$, this means that $\mu\left(A \cap B_{r}\right)>$ 
0. Fix $x \in A \cap B_{r}$ for some $r \in(0,1)$, then

$$
\begin{gathered}
(C-1) \sum_{i=1}^{+\infty}\left(x, v_{i}\right)_{X}^{2}+\frac{4}{(x, x)_{X}} \sum_{i=1}^{+\infty} \frac{\left(x, v_{i}\right)_{X}^{2}}{4^{i}}-\frac{8}{(x, x)_{X}^{2}}\left(\sum_{i=1}^{+\infty} \frac{\left(x, v_{i}\right)_{X}^{2}}{2^{i}}\right)^{2} \leq \\
\leq(C-1) \sum_{i=1}^{+\infty}\left(x, v_{i}\right)_{X}^{2}+\frac{4}{(x, x)_{X}} \sum_{i=1}^{+\infty} \frac{\left(x, v_{i}\right)_{X}^{2}}{4^{i}}-\frac{8}{(x, x)_{X}^{2}}\left(\sum_{i=1}^{+\infty} \frac{\left(x, v_{i}\right)_{X}^{2}}{4^{i}}\right)^{2} \leq \\
\leq(C-1)\|x\|_{X}^{2}+4-2\|x\|_{X}^{4} \frac{8}{\|x\|_{X}^{4}}=(C-1)\|x\|_{X}^{2}-12
\end{gathered}
$$

Observe that if $C>1$ and $x \in A \cap B_{\left(6(C-1)^{-1}\right)^{\frac{1}{2}}}$, then $(4.7) \leq-6 \mu$-a.e., a contradiction. Thus our example does not satisfy (4.4).

Observe that if $\Phi \in W^{1,2 \alpha}(X, \mu ; H)$ with $\alpha>1$, then $\Phi \in D\left(\operatorname{div}_{\nu}\right)$. Indeed let $\varphi_{n}=\left\langle\Phi, e_{n}\right\rangle_{H}$ for every $n \in \mathbb{N}$, then by $(4.6)$

$$
\begin{gathered}
\sum_{i=1}^{+\infty} \sum_{j=1}^{+\infty}\left(\delta_{i j}-\partial_{i} \partial_{j} \log w(x)\right) \varphi_{i} \varphi_{j}= \\
=\sum_{i=1}^{+\infty} \sum_{j=1}^{+\infty}\left(\delta_{i j}-4 \delta_{i j} \frac{\sqrt{\lambda_{i} \lambda_{j}}}{(x, x)_{X}}+8 \frac{\sqrt{\lambda_{i} \lambda_{j}}\left(x, v_{i}\right)_{X}\left(x, v_{j}\right)_{X}}{(x, x)_{X}^{2}}\right) \varphi_{i} \varphi_{j}= \\
=\sum_{i=1}^{+\infty} \varphi_{i}^{2}-4 \sum_{i=1}^{+\infty} \frac{\lambda_{i} \varphi_{i}^{2}}{(x, x)_{X}}+\frac{8}{(x, x)_{X}^{2}}\left(\sum_{i=1}^{+\infty} \sqrt{\lambda_{i}} \varphi_{i}\left(x, v_{i}\right)_{X}\right)^{2} \leq \\
\leq|\Phi|_{H}^{2}+8 \frac{\left(x, \sum_{i=1}^{+\infty} \varphi_{i} e_{i}\right)_{X}^{2}}{(x, x)_{X}^{2}}=|\Phi|_{H}^{2}+8 \frac{(x, \Phi)_{X}^{2}}{(x, x)_{X}^{2}} \leq(*)|\Phi|_{H}^{2}+8 \frac{\|\Phi\|_{X}^{2}}{(x, x)_{X}} \leq|\Phi|_{H}^{2}+8 K \frac{|\Phi|_{H}^{2}}{(x, x)_{X}},
\end{gathered}
$$

where $(*)$ follows by the Schwarz inequality and $\|h\|_{X} \leq K|h|_{H}$ for every $h \in H$. We can now integrate both terms of the inequality

$$
\begin{gathered}
\int_{X} \sum_{i=1}^{+\infty} \sum_{j=1}^{+\infty}\left(\delta_{i j}-\partial_{i} \partial_{j} \log w(x)\right) \varphi_{i} \varphi_{j} d \nu \leq \int_{X}\left(|\Phi|_{H}^{2}+8 K \frac{|\Phi|_{H}^{2}}{(x, x)_{X}}\right) d \nu= \\
=\int_{X}|\Phi|_{H}^{2}(x, x)_{X}^{2} d \mu+8 K \int_{X}|\Phi|_{H}^{2}(x, x)_{X} d \mu .
\end{gathered}
$$

By the Hölder inequality and Fernique's theorem (see [7, Theorem 2.8.5]) there exists a constant $\bar{K}$ such that

$$
\int_{X} \sum_{i=1}^{+\infty} \sum_{j=1}^{+\infty}\left(\delta_{i j}-\partial_{i} \partial_{j} \log w(x)\right) \varphi_{i} \varphi_{j} d \nu \leq \bar{K}\|\Phi\|_{\mathrm{L}^{2 \alpha}(X, \mu ; H)}^{2}
$$

Fix now a field of the following form: $\Phi(x)=\sum_{i=1}^{n} \varphi_{i}(x) e_{i}$ with $\varphi_{i} \in \mathscr{F} \mathscr{C}_{b}^{\infty}(X)$ for every $i=1, \ldots, n$. Following the same steps of the proof of Proposition 4.3 we rewrite (4.5) as

$$
\int_{X}\left(\operatorname{div}_{\nu} \Phi\right)^{2} d \nu \leq \bar{K}\|\Phi\|_{L^{2 \alpha}(X, \mu ; H)}^{2}+\int_{X}\left\|\nabla_{H} \Phi(x)\right\|_{\mathcal{H}}^{2} d \nu(x) \leq \max \{\bar{K}, 1\}\|\Phi\|_{W^{1,2 \alpha}(X, \mu ; H)}^{2} .
$$

By the same argument used at the end of the proof of Proposition 4.3 and by (2) of Proposition 3.5 (since it implies that $W^{1,2 \alpha}(X, \mu ; H) \subseteq \mathrm{L}^{1}(X, \nu ; X)$ for every $\left.\alpha>1\right)$, we obtain that $W^{1,2 \alpha}(X, \mu ; H) \subseteq$ $D\left(\operatorname{div}_{\nu}\right)$ for every $\alpha>1$.

Since in several pratical examples the vector field we are interested in belongs to $W^{1, q}(X, \mu ; H)$ for some $q>1$, the following proposition might be useful. 
Proposition 4.5. Let $\Phi \in W^{1, q}(X, \mu ; H)$ for some $q \geq \frac{s^{\prime} t}{t-s^{\prime}}$. Then $\operatorname{div}_{\nu} \Phi$ exists and it belongs to $\mathrm{L}^{p}(X, \nu)$ for every $p \in\left[1, \frac{q t}{s^{\prime}(q+t)}\right)$. Furthermore

$$
\operatorname{div}_{\nu} \Phi=\operatorname{div}_{\mu} \Phi+\left\langle\Phi, \nabla_{H} \log w\right\rangle_{H}
$$

and $\left\|\operatorname{div}_{\nu} \Phi\right\|_{\mathrm{L}^{p}(X, \nu)} \leq C_{p}\|\Phi\|_{W^{1, q}(X, \mu ; H)}$ for some $C_{p}>0$. Finally if $\Phi \in W^{1, q}(X, \mu ; H)$ for every $q>1$, then $\operatorname{div}_{\nu} \Phi \in \mathrm{L}^{r}(X, \nu)$ for every $r \in\left[1, t / s^{\prime}\right)$.

Proof. By the Hölder inequality $\left\langle\Phi, \nabla_{H} \log w\right\rangle_{H} \in \mathrm{L}^{\frac{q t}{q+t}}(X, \mu)$ and by [7, Proposition 5.8.8] $\operatorname{div}_{\mu} \Phi \in$ $\mathrm{L}^{q}(X, \mu)$. By (2) of Proposition 3.5 both $\operatorname{div}_{\mu} \Phi$ and $\left\langle\Phi, \nabla_{H} \log w\right\rangle_{H}$ belong to $\mathrm{L}^{p}(X, \nu)$ for every $p \in\left[1, \frac{q t}{s^{\prime}(q+t)}\right]$. Let $f \in \mathscr{F} \mathscr{C}_{b}^{\infty}(X)$ and $\left(w_{n}\right)_{n \in \mathbb{N}} \subseteq \mathscr{F} \mathscr{C}_{b}^{\infty}(X)$ be a sequence of positive functions which converges to $w$ pointwise and in $W^{1, s}(X, \mu)$ (so that also $\log w_{n}$ converges to $\log w$ both pointwise and in $\left.W^{1, t}(X, \mu)\right)$, then

$$
\begin{gathered}
\int_{X} f\left(\operatorname{div}_{\mu} \Phi+\left\langle\Phi, \nabla_{H} \log w_{n}\right\rangle_{H}\right) w_{n} d \mu=\int_{X}\left(f w_{n}\right) \operatorname{div}_{\mu} \Phi d \mu+\int_{X} f\left\langle\Phi, \nabla_{H} \log w_{n}\right\rangle_{H} w_{n} d \mu= \\
=-\int_{X}\left\langle\nabla_{H}\left(f w_{n}\right), \Phi\right\rangle_{H} d \mu+\int_{X} f\left\langle\Phi, \nabla_{H} \log w_{n}\right\rangle_{H} w_{n} d \mu= \\
=-\int_{X}\left\langle\nabla_{H} f, \Phi\right\rangle_{H} w_{n} d \mu-\int_{X} f\left\langle\nabla_{H}\left(w_{n}\right), \Phi\right\rangle_{H} d \mu+\int_{X} f\left\langle\Phi, \nabla_{H} \log w_{n}\right\rangle_{H} w_{n} d \mu= \\
=-\int_{X}\left\langle\nabla_{H} f, \Phi\right\rangle_{H} w_{n} d \mu-\int_{X} f\left\langle\nabla_{H}\left(\log w_{n}\right), \Phi\right\rangle_{H} w_{n} d \mu+\int_{X} f\left\langle\Phi, \nabla_{H} \log w_{n}\right\rangle_{H} w_{n} d \mu= \\
=-\int_{X}\left\langle\nabla_{H} f, \Phi\right\rangle_{H} w_{n} d \mu .
\end{gathered}
$$

Letting $n \rightarrow+\infty$ we obtain that $\operatorname{div}_{\nu} \Phi=\operatorname{div}_{\mu} \Phi+\left\langle\Phi, \nabla_{H} \log w\right\rangle_{H}$. The inequality

$$
\left\|\operatorname{div}_{\nu} \Phi\right\|_{L^{p}(X, \nu)} \leq C_{p}\|\Phi\|_{W^{1, q}(X, \mu ; H)}
$$

follows by a standard Hölder inequality application and [7, Proposition 5.8.8].

\section{Sobolev Spaces on Sublevel Sets}

Let $G$ be a function satisfying Hypothesis 1.2. We are interested in Sobolev spaces on sets of the form $G^{-1}(-\infty, 0)$. Via a standard argument it is possible to prove that $\operatorname{Lip}\left(G^{-1}(-\infty, 0)\right)$ is a dense subspace in $\mathrm{L}^{p}\left(G^{-1}(-\infty, 0), \nu\right)$ (see [4]). Whenever $p \geq \frac{t}{t-s^{\prime}}$ it is possible to define

$$
\nabla_{H}^{0}: \operatorname{Lip}\left(G^{-1}(-\infty, 0)\right) \longrightarrow \mathrm{L}^{p}\left(G^{-1}(-\infty, 0), \nu ; H\right)
$$

in the following way: for every $\varphi \in \operatorname{Lip}\left(G^{-1}(-\infty, 0)\right)$, with Lipschitz constant $L_{\varphi}$, consider the McShane extension (see [18])

$$
\varphi_{M}(x)=\sup \left\{\varphi(y)+L_{\varphi}\|x-y\|_{X} \mid G(y)<0\right\} .
$$

It is well known that $\varphi_{M} \in \operatorname{Lip}(X) \subseteq W^{1, q}(X, \nu)$ for some $q \geq \frac{t}{t-s^{\prime}}$ ((2) of Proposition 3.5 and [7, Theorem 5.11.2]). Let

$$
\nabla_{H}^{0} \varphi:=\nabla_{H} \varphi_{M}
$$

Proposition 5.1. Let $p \geq \frac{t}{t-s^{\prime}}$. The operator

$$
\nabla_{H}^{0}: \operatorname{Lip}\left(G^{-1}(-\infty, 0)\right) \rightarrow L^{p}\left(G^{-1}(-\infty, 0), \nu ; H\right)
$$

is closable in $\mathrm{L}^{p}\left(G^{-1}(-\infty, 0), \nu\right)$.

Proof. Let $\left(f_{k}\right)_{k \in \mathbb{N}} \subseteq \operatorname{Lip}\left(G^{-1}(-\infty, 0)\right)$ such that

$$
\begin{aligned}
& \lim _{k \rightarrow+\infty} f_{k}=0 \quad \text { in } L^{p}\left(G^{-1}(-\infty, 0), \nu\right) ; \\
& \lim _{k \rightarrow+\infty} \nabla_{H}^{0} f_{k}=\Phi \quad \text { in } L^{p}\left(G^{-1}(-\infty, 0), \nu ; H\right) .
\end{aligned}
$$


We want to prove that

$$
\int_{G^{-1}(-\infty, 0)}\left\langle\Phi(x), e_{i}\right\rangle u(x) d \nu(x)=0
$$

for every $i \in \mathbb{N}$ and $u \in \mathscr{F} \mathscr{C}_{b}^{\infty}(X)$. Let $\eta: \mathbb{R} \rightarrow \mathbb{R}$ a smooth function such that $\|\eta\|_{\infty} \leq 1,\left\|\eta^{\prime}\right\|_{\infty} \leq 2$ and

$$
\eta(\xi)= \begin{cases}0 & \xi \geq-1 \\ 1 & \xi \leq-2\end{cases}
$$

Let $\eta_{n}(\xi):=\eta(n \xi)$ and $u_{n}(x)=u(x) \eta_{n}(G(x))$. Observe that $u_{n}$ converges pointwise $\nu$-a.e. to $u$ on $G^{-1}(-\infty, 0)$ and $\left|u_{n}\right| \leq|u| \nu$-a.e., then by Lebesgue's dominated convergence theorem

$$
\lim _{n \rightarrow+\infty} \int_{G^{-1}(-\infty, 0)}\left\langle\Phi(x), e_{i}\right\rangle u_{n}(x) d \nu(x)=\int_{G^{-1}(-\infty, 0)}\left\langle\Phi(x), e_{i}\right\rangle u(x) d \nu(x) .
$$

By (5) of Proposition 3.5, for every $n \in \mathbb{N}$ we have $u_{n} \in W^{1, r}(X, \nu)$, for every $r \geq \frac{t}{t-s^{\prime}}$, and by Proposition 3.6

$$
\partial_{i} u_{n}(x)=\partial_{i} u(x) \eta_{n}(G(x))+u(x) \eta_{n}^{\prime}(G(x)) \partial_{i} G(x) .
$$

Observe that

$$
\int_{X} u_{n} \partial_{i} f_{k} d \nu=\int_{X} f_{k} u_{n}\left(\widehat{e}_{i}-\partial_{i} \log w\right) d \nu-\int_{X} f_{k} \partial_{i} u\left(\eta_{n} \circ G\right) d \nu-\int_{X} f_{k} u\left(\eta^{\prime} \circ G\right) \partial_{i} G d \nu,
$$

and the following estimates holds:

$$
\begin{gathered}
\int_{X}\left|\partial_{i} f_{k} u_{n}-\left\langle\Phi, e_{i}\right\rangle_{H} u\right| d \nu \leq \int_{X}\left|\partial_{i} f_{k}\right|\left|u_{n}-u\right| d \nu+\int_{X}\left|\partial_{i} f_{k}-\left\langle\Phi, e_{i}\right\rangle_{H}\right||u| d \nu \leq \\
\leq\left(\int_{X}\left|\partial_{i} f_{k}\right|^{p} d \nu\right)^{\frac{1}{p}}\left(\int_{X}\left|u_{n}-u\right|^{p^{\prime}} d \nu\right)^{\frac{1}{p^{\prime}}}+\left(\int_{X}\left|\partial_{i} f_{k}-\left\langle\Phi, e_{i}\right\rangle_{H}\right|^{p} d \nu\right)^{\frac{1}{p}}\left(\int_{X}|u|^{p^{\prime}} d \nu\right)^{\frac{1}{p^{\prime}}},
\end{gathered}
$$

this means $\lim _{n \rightarrow+\infty} \lim _{k \rightarrow+\infty} \int_{X} u_{n} \partial_{i} f_{k} d \nu=\int_{G^{-1}(-\infty, 0)}\left\langle\Phi, e_{i}\right\rangle u d \nu$. Furthermore for every $n \in \mathbb{N}$ we get

$$
\begin{gathered}
\int_{X}\left|f_{k} \partial_{i} u\left(\eta_{n} \circ G\right)\right| d \nu \leq \int_{X}\left|f_{k} \partial_{i} u\right| d \nu \leq\left(\int_{X}\left|f_{k}\right|^{p} d \nu\right)^{\frac{1}{p}}\left(\int_{X}\left|\partial_{i} u\right|^{p^{\prime}} d \nu\right)^{\frac{1}{p^{\prime}}} \stackrel{k \rightarrow+\infty}{\longrightarrow} 0 ; \\
\int_{X}\left|f_{k} u\left(\eta_{n}^{\prime} \circ G\right) \partial_{i} G\right| d \nu \leq\|u\|_{\infty}\left(\int_{X}\left|f_{k}\right|^{p} d \nu\right)^{\frac{1}{p}}\left(\int_{X}\left|\partial_{i} G\right|^{p^{\prime} s^{\prime}} d \mu\right)^{\frac{1}{p^{\prime} s^{\prime}}}\left(\int_{X} w^{s} d \mu\right)^{\frac{1}{p^{\prime} s}} \stackrel{k \rightarrow+\infty}{\longrightarrow} 0,
\end{gathered}
$$

where the last limit follows by to Hypothesis 1.2;

$$
\begin{gathered}
\int_{X}\left|f_{k} u_{n} \widehat{e}_{i}\right| d \nu \leq\|u\|_{\infty}\left(\int_{X}\left|f_{k}\right|^{p} d \nu\right)^{\frac{1}{p}}\left(\int_{X}\left|\widehat{e}_{i}\right|^{p^{\prime}} d \nu\right)^{\frac{1}{p^{\prime}}} \stackrel{k \rightarrow+\infty}{\longrightarrow} 0 \\
\int_{X}\left|f_{k} u_{n} \partial_{i} \log w\right| d \nu \leq\|u\|_{\infty}\left(\int_{X}\left|f_{k}\right|^{p} d \nu\right)^{\frac{1}{p}}\left(\int_{X}|w|^{s} d \mu\right)^{\frac{1}{p^{\prime} s}}\left(\int_{X}\left|\partial_{i} \log w\right|^{p^{\prime} s^{\prime}} d \mu\right)^{\frac{1}{p^{\prime} s^{\prime}}} \stackrel{k \rightarrow+\infty}{\longrightarrow} 0,
\end{gathered}
$$

and the last limit exists whenever $p^{\prime} s^{\prime} \leq t$.

Definition 5.2 (Weighted Sobolev space on sublevel sets). Let $p \geq \frac{t}{t-s^{\prime}}$. We denote by $W^{1, p}\left(G^{-1}(-\infty, 0), \nu\right)$ the domain of the closure of the operator $\nabla_{H}^{0}$ (which we will denote by the symbol $\nabla_{H}$ ) in $\mathrm{L}^{p}\left(G^{-1}(-\infty, 0), \nu\right)$. It is a Banach space with the graph norm

$$
\|f\|_{W^{1, p}\left(G^{-1}(-\infty, 0), \nu\right)}=\left(\int_{G^{-1}(-\infty, 0)}|f(x)|^{p} d \nu(x)\right)^{\frac{1}{p}}+\left(\int_{G^{-1}(-\infty, 0)}\left|\nabla_{H} f(x)\right|_{H}^{p} d \nu(x)\right)^{\frac{1}{p}} .
$$

The following equalities are what will allow us to define the trace operator in the following section. 
Proposition 5.3. Let $k \in \mathbb{N}$. Then for every $\varphi \in \operatorname{Lip}_{b}\left(G^{-1}(-\infty, 0)\right)$ and $G$ satisfying Hypothesis 1.2, we have

$$
\int_{G^{-1}(-\infty, 0)}\left(\partial_{k} \varphi+\varphi \partial_{k} \log w-\varphi \widehat{e}_{k}\right) d \nu=\int_{G^{-1}(0)}\left(\frac{\varphi \partial_{k} G}{\left|\nabla_{H} G\right|_{H}}\right)_{\left.\right|_{G^{-1}(0)}} w d \rho .
$$

Proof. The proof is the same as [10, Equation (1.1), proof in Proposition 4.1].

Proposition 5.4. Let $q \geq 1$. Then for every $\varphi \in \operatorname{Lip}_{b}\left(G^{-1}(-\infty, 0)\right)$ and $G$ satisfying Hypothesis 1.2, we have

$$
\int_{G^{-1}(-\infty, 0)}\left(q \varphi|\varphi|^{q-2}\left\langle\nabla_{H} \varphi, \nabla_{H} G\right\rangle_{H}-|\varphi|^{q} \operatorname{div}_{\nu} \nabla_{H} G\right) d \nu=\int_{G^{-1}(0)}\left(|\varphi|^{q}\left|\nabla_{H} G\right|_{H}\right)_{\left.\right|_{G^{-1}(0)}} w d \rho,
$$

and

$$
\int_{G^{-1}(-\infty, 0)}\left(q \varphi|\varphi|^{q-2}\left\langle\nabla_{H} \varphi, \frac{\nabla_{H} G}{\left|\nabla_{H} G\right|_{H}}\right\rangle_{H}+\operatorname{div}_{\nu} \frac{\nabla_{H} G}{\left|\nabla_{H} G\right|_{H}}|\varphi|^{q}\right) d \nu=\int_{G^{-1}(0)}\left(|\varphi|^{q}\right)_{\left.\right|_{G^{-1}(0)}} w d \rho .
$$

Proof. The proof is the same as [10, Proposition 4.1].

\section{Traces of Sobolev functions on SUblevel Sets}

Throughout this section we will denote by $G$ a function satisfying Hypothesis 1.2. The following result is fundamental for the definition of the trace operator.

Proposition 6.1. Let $p \geq \frac{t}{t-s^{\prime}}$. The following holds:

(1) if $\left(\varphi_{n}\right)_{n \in \mathbb{N}} \subseteq \operatorname{Lip}_{b}\left(G^{-1}(-\infty, 0)\right)$ is a Cauchy sequence in $W^{1, p}\left(G^{-1}(-\infty, 0), \nu\right)$, then $\left(\varphi_{n_{\left.\right|_{G}-1}(0)}\right)$ is a Cauchy sequence in $\mathrm{L}^{q}\left(G^{-1}(0), w \rho\right)$ for every $1 \leq q \leq p \frac{t-s^{\prime}}{t}$;

(2) if $\left(\varphi_{n}\right)_{n \in \mathbb{N}},\left(\psi_{n}\right)_{n \in \mathbb{N}} \subseteq \operatorname{Lip}_{b}\left(G^{-1}(-\infty, 0)\right)$ converge to $\varphi$ in $W^{1, p}\left(G^{-1}(-\infty, 0), \nu\right)$, then $\left(\varphi_{n_{\left.\right|_{G}-1(0)}}\right)$ and $\left(\psi_{n_{G^{-1}(0)}}\right)$ converge to the same element in $\mathrm{L}^{q}\left(G^{-1}(0), w \rho\right)$ for every $1 \leq q \leq p \frac{t-s^{\prime}}{t}$.

Proof. Let $\left(\varphi_{n}\right)_{n \in \mathbb{N}} \subseteq \operatorname{Lip}_{b}\left(G^{-1}(-\infty, 0)\right)$ be a Cauchy sequence in $W^{1, p}\left(G^{-1}(-\infty, 0), \nu\right)$. By Proposition 5.4 we have for every $q \in\left[1, p \frac{t-s^{\prime}}{t}\right]$

$$
\begin{aligned}
& \int_{G^{-1}(0)}\left|\varphi_{n}-\varphi_{m}\right|^{q} w d \rho=\int_{G^{-1}(-\infty, 0)}\left(q\left|\varphi_{n}-\varphi_{m}\right|^{q-2}\left(\varphi_{n}-\varphi_{m}\right)\left\langle\nabla_{H}\left(\varphi_{n}-\varphi_{m}\right), \frac{\nabla_{H} G}{\left|\nabla_{H} G\right|_{H}}\right\rangle_{H}\right) d \nu+ \\
&+\int_{G^{-1}(-\infty, 0)}\left(\operatorname{div}_{\nu} \frac{\nabla_{H} G}{\left|\nabla_{H} G\right|_{H}}\left|\varphi_{n}-\varphi_{m}\right|^{q}\right) d \nu \stackrel{n, m \rightarrow+\infty}{\longrightarrow} 0 .
\end{aligned}
$$

All the convergences are assured by Proposition 4.5. Thus (1) is proved.

Let $\left(\varphi_{n}\right)_{n \in \mathbb{N}},\left(\psi_{n}\right)_{n \in \mathbb{N}} \subseteq \operatorname{Lip}_{b}\left(G^{-1}(-\infty, 0)\right)$ satisfying $(2)$ and let $\varphi_{\infty}$ the limit of $\left(\varphi_{n_{\left.\right|^{-1}}(0)}\right)$ in $\mathrm{L}^{q}\left(G^{-1}(0), w \rho\right)$ for every $1 \leq q \leq p \frac{t-s^{\prime}}{t}$ (it exists by (1)). By Proposition 5.4 we have for every $q \in\left[1, p \frac{t-s^{\prime}}{t}\right]$

$$
\begin{aligned}
& \int_{G^{-1}(0)}\left|\psi_{n}-\varphi_{\infty}\right|^{q} w d \rho \leq 2^{q-1}\left(\int_{G^{-1}(0)}\left|\psi_{n}-\varphi_{n}\right|^{q} w d \rho+\int_{G^{-1}(0)}\left|\varphi_{n}-\varphi_{\infty}\right|^{q} w d \rho\right)= \\
& \quad=2^{q-1}\left(\int_{G^{-1}(-\infty, 0)}\left(q\left|\psi_{n}-\varphi_{n}\right|^{q-2}\left(\psi_{n}-\varphi_{n}\right)\left\langle\nabla_{H}\left(\psi_{n}-\varphi_{n}\right), \frac{\nabla_{H} G}{\left|\nabla_{H} G\right|_{H}}\right\rangle_{H}\right) d \nu+\right. \\
& \left.+\int_{G^{-1}(-\infty, 0)}\left(\operatorname{div}_{\nu} \frac{\nabla_{H} G}{\left|\nabla_{H} G\right|_{H}}\left|\psi_{n}-\varphi_{n}\right|^{q}\right) d \nu+\int_{G^{-1}(0)}\left|\varphi_{n}-\varphi_{\infty}\right|^{q} w d \rho\right) \stackrel{n, m \rightarrow+\infty}{\longrightarrow} 0 .
\end{aligned}
$$

Thus (2) is proved.

We are now able to define the trace of a Sobolev function. 
Definition 6.2. Let $p \geq \frac{t}{t-s^{\prime}}$. If $\varphi \in W^{1, p}\left(G^{-1}(-\infty, 0), \nu\right)$ we define the trace of $\varphi$ on $G^{-1}(0)$ as follows:

$$
\operatorname{Tr}_{G^{-1}(0)} \varphi=\lim _{n \rightarrow+\infty} \varphi_{n_{G^{-1}(0)}} \quad \text { in } \mathrm{L}^{q}\left(G^{-1}(0), w \rho\right) \text { for every } q \in\left[1, p \frac{t-s^{\prime}}{t}\right],
$$

where $\left(\varphi_{n}\right)_{n \in \mathbb{N}}$ is any sequence in $\operatorname{Lip}_{b}\left(G^{-1}(-\infty, 0)\right)$ which converges in $W^{1, p}\left(G^{-1}(-\infty, 0), \nu\right)$ to $\varphi$. By Proposition 6.1 the definition does not depend on the choice of the sequence $\left(\varphi_{n}\right)_{n \in \mathbb{N}}$ in $\operatorname{Lip}_{b}\left(G^{-1}(-\infty, 0)\right)$ approximating $\varphi$ in $W^{1, p}\left(G^{-1}(-\infty, 0), \nu\right)$.

Observe that by [10, Proposition 4.8] $w \rho=w_{\left.\right|_{G^{-1}(0)}} \rho$. An obvious consequence of the definition is the following Corollary.

Corollary 6.3. Let $p \geq \frac{t}{t-s^{\prime}}$. The operator

$$
\operatorname{Tr}_{G^{-1}(0)}: W^{1, p}\left(G^{-1}(-\infty, 0), \nu\right) \longrightarrow \mathrm{L}^{q}(X, w \rho)
$$

is continuous for every $q \in\left[1, p \frac{t-s^{\prime}}{t}\right]$.

Proposition 6.4. Let $a>1$ and $b>1$. If $\frac{a b}{a+b} \geq \frac{t}{t-s^{\prime}}$, then for every $\varphi \in W^{1, a}(X, \nu)$ and $\psi \in$ $W^{1, b}(X, \nu)$ we have

$$
\operatorname{Tr}_{G^{-1}(0)}(\varphi \psi)=\operatorname{Tr}_{G^{-1}(0)}(\varphi) \operatorname{Tr}_{G^{-1}(0)}(\psi) \quad \rho \text {-a.e. }
$$

Proof. Let $\left(\varphi_{n}\right)_{n \in \mathbb{N}},\left(\psi_{n}\right)_{n \in \mathbb{N}} \subseteq \operatorname{Lip}_{b}\left(G^{-1}(-\infty, 0)\right)$ such that

$$
\begin{array}{ll}
\lim _{n \rightarrow+\infty} \varphi_{n}=\varphi & \text { in } W^{1, a}\left(G^{-1}(-\infty, 0), \nu\right) ; \\
\lim _{n \rightarrow+\infty} \psi_{n}=\psi & \text { in } W^{1, b}\left(G^{-1}(-\infty, 0), \nu\right) .
\end{array}
$$

By a standard argument we know $\varphi_{n} \psi_{n} \in \operatorname{Lip}_{b}\left(G^{-1}(-\infty, 0)\right)$, for every $n \in \mathbb{N}$, and

$$
\lim _{n \rightarrow+\infty} \varphi_{n} \psi_{n}=\varphi \psi \quad \text { in } W^{1, \frac{a b}{a+b}}\left(G^{-1}(-\infty, 0), \nu\right) .
$$

So we have

$$
\operatorname{Tr}_{G^{-1}(0)}(\varphi \psi)=\lim _{n \rightarrow+\infty} \varphi_{n_{G^{-1}(0)}} \psi_{n_{G^{-1}}(0)} \quad \text { in } \mathrm{L}^{q}\left(G^{-1}(0), w \rho\right) \text { for every } q \in\left[1, \frac{a b}{a+b} \frac{t-s^{\prime}}{t}\right] .
$$

Using Hölder inequality we get

$$
\int_{X}\left|\varphi_{n_{\left.\right|_{G}-1}(0)} \psi_{n_{\left.\right|_{G^{-1}}(0)}}-\operatorname{Tr}_{G^{-1}(0)}(\varphi) \operatorname{Tr}_{G^{-1}(0)}(\psi)\right| w d \rho \stackrel{n \rightarrow+\infty}{\longrightarrow} 0 .
$$

So $\operatorname{Tr}_{G^{-1}(0)}(\varphi \psi)=\operatorname{Tr}_{G^{-1}(0)}(\varphi) \operatorname{Tr}_{G^{-1}(0)}(\psi) \rho$-a.e.

Proposition 6.5. Let $p \geq \frac{t}{t-s^{\prime}}$. For every $\varphi \in W^{1, p}\left(G^{-1}(-\infty, 0), \nu\right)$ we have

$$
\operatorname{Tr}_{G^{-1}(0)} \varphi(x)=\bar{\varphi}_{\left.\right|_{G^{-1}(0)}}(x) \quad \rho \text {-a.e. }
$$

for every $(1, p)$-precise version $\bar{\varphi}$ of $\varphi$.

Proof. Let $\left(\varphi_{n}\right)_{n \in \mathbb{N}}$ a sequence of bounded Lipschitz functions defined on $G^{-1}(-\infty, 0)$ which satisties the condition of the Definition 6.2. By Proposition 5.4 we get

$$
\begin{gathered}
\int_{G^{-1}(0)}\left|\bar{\varphi}_{\left.\right|_{G^{-1}(0)}}-\varphi_{n}\right|\left|\nabla_{H} G\right|_{H} w d \rho= \\
=\int_{G^{-1}(-\infty, 0)}\left(\operatorname{sign}\left(\bar{\varphi}-\varphi_{n}\right)\left\langle\nabla_{H}\left(\bar{\varphi}-\varphi_{n}\right), \nabla_{H} G\right\rangle_{H}-\left|\bar{\varphi}-\varphi_{n}\right| \operatorname{div}_{\nu} \nabla_{H} G\right) d \nu .
\end{gathered}
$$

Letting $n \rightarrow+\infty$ we get $\operatorname{Tr}_{G^{-1}(0)} \varphi=\bar{\varphi}_{\left.\right|_{G^{-1}(0)}} \rho$-a.e.

We are now in a position to prove Theorem 1.3. 
Proof of Theorem 1.3. The statement follows by Proposition 5.3 and Proposition 6.5. The furthermore part follows by Proposition 6.4.

Using the same argument we can extend Proposition 5.4 to functions in $W^{1, p}\left(G^{-1}(-\infty, 0), \nu\right)$.

Proposition 6.6. Let $p \geq \frac{t}{t-s^{\prime}}$ and $1 \leq q \leq p \frac{t-s^{\prime}}{t}$. If $\varphi \in W^{1, p}\left(G^{-1}(-\infty, 0), \nu\right)$ then

$$
\begin{gathered}
\int_{G^{-1}(-\infty, 0)}\left(q \varphi|\varphi|^{q-2}\left\langle\nabla_{H} \varphi, \nabla_{H} G\right\rangle_{H}-|\varphi|^{q} \operatorname{div}_{\nu} \nabla_{H} G\right) d \nu= \\
=\int_{G^{-1}(0)} \operatorname{Tr}_{G^{-1}(0)}|\varphi|^{q} \operatorname{Tr}_{G^{-1}(0)}\left|\nabla_{H} G\right|_{H} w d \rho
\end{gathered}
$$

and

$$
\int_{G^{-1}(-\infty, 0)}\left(q \varphi|\varphi|^{q-2}\left\langle\nabla_{H} \varphi, \frac{\nabla_{H} G}{\left|\nabla_{H} G\right|_{H}}\right\rangle+\operatorname{div}_{\nu} \frac{\nabla_{H} G}{\left|\nabla_{H} G\right|_{H}}|\varphi|^{q}\right) d \nu=\int_{G^{-1}(0)}\left(\operatorname{Tr}_{G^{-1}(0)}|\varphi|^{q}\right) w d \rho .
$$

\section{EXAMPLES}

In this section we show how our results may be applied to some explicit examples. Recall that by $\partial_{i} f$ we denote the partial derivative of $f$ along the direction $e_{i} \in H$ (see Section 2). We will be interested in two types of surfaces:

Unit sphere: Let $S(x)=\|x\|_{X}-1$. We will prove that $S$ satisfies Hypothesis 1.2 in our examples.

Hyperplanes: Let $f \in X^{*} \backslash\{0\}$ and $G_{f}(x)=f(x)$. Observe that $\partial_{i} G_{f}(x)=f\left(e_{i}\right)$ for every $i \in \mathbb{N}$ and $\partial_{i} \partial_{j} G_{f}(x)=0$ for every $i, j \in \mathbb{N}$. Furthermore

$$
G_{f} \in \bigcap_{p>1} W^{2, p}(X, \mu) \quad \text { and } \quad \frac{1}{\left|\nabla_{H} G_{f}\right|_{H}} \in \bigcap_{p>1} \mathrm{~L}^{p}(X, \mu) .
$$

Thus $G_{f}$ satifies Hypothesis 1.2 , for every $f \in X^{*} \backslash\{0\}$.

7.1. A Gaussian-type weight in Hilbert spaces. Let $X$ be a separable Hilbert space endowed with a nondegenerate centered Gaussian measure $\mu$, with covariance $Q$. Fix an orthonormal basis $\left(v_{n}\right)_{n \in \mathbb{N}}$ of $X$ of eigenvectors of $Q$, i.e. $Q v_{k}=\lambda_{k} v_{k}$, and the corresponding orthonormal basis of $H=Q^{\frac{1}{2}}(X)$ is $\left\{e_{n}:=\sqrt{\lambda_{n}} v_{n}\right\}_{n \in \mathbb{N}}$.

Let $w_{\lambda}(x)=e^{\lambda(x, x)_{X}}$ for $\lambda \in \mathbb{R}$. Easy calculation gives

$$
\partial_{i} w_{\lambda}(x)=2 \lambda\left(x, e_{i}\right)_{X} e^{\lambda(x, x)_{X}}, \quad \partial_{i} \log w_{\lambda}(x)=2 \lambda\left(x, e_{i}\right)_{X}, \quad \partial_{j} \partial_{i} \log w_{\lambda}(x)=2 \lambda\left(e_{j}, e_{i}\right)_{X} .
$$

Let

$$
\alpha:=\sup \left\{\eta>0 \mid \int_{X} e^{\eta(x, x)_{X}} d \mu(x)<+\infty\right\} .
$$

By Fernique's theorem (see [7, Theorem 2.8.5]) the set $\left\{\eta>0 \mid \int_{X} e^{\eta(x, x)_{X}} d \mu(x)<+\infty\right\}$ is not empty and $\alpha$ is strictly positive. Furthermore

$$
\begin{gathered}
\int_{X} e^{\eta(x, x)_{X}} d \mu(x)=\int_{X} e^{\eta \sum_{i=1}^{+\infty}\left(x, v_{i}\right)_{X}^{2}} d \mu(x)=\lim _{n \rightarrow+\infty} \int_{X} e^{\eta \sum_{i=1}^{n}\left(x, v_{i}\right)_{X}^{2}} d \mu(x)= \\
=\lim _{n \rightarrow+\infty} \prod_{i=1}^{n} \sqrt{\frac{\lambda_{i}}{2 \pi}} \int_{\mathbb{R}} e^{\left(\eta-\frac{\lambda_{i}}{2}\right) \xi^{2}} d \xi
\end{gathered}
$$

and the last limit diverges if $\eta \geq 2 \lambda_{1}$. Thus $0<\alpha<2 \lambda_{1}$ and $w_{\lambda} \in W^{1, s}(X, \mu)$ for every $2 \lambda<\alpha$ whenever $\lambda(s+1) \leq \alpha$. Furthermore $\log w_{\lambda} \in W^{2, t}(X, \mu)$ for every $t>1$ and $2 \lambda<\alpha$. In both cases it is possible to define $W^{1, p}(X, \nu)$ for every $p>1$ (see Definition 3.3).

The above observation gives also that $S$ satisfies Hypothesis 1.2. In this case $w_{\lambda} \rho=e^{\lambda} \rho$, so all the remarks about continuity of the trace operator in $\mathrm{L}^{p}\left(S^{-1}(0), \rho\right)$ stated in [10, Section 5.3] still hold. 
By Corollary 6.3, the trace operator $\operatorname{Tr}_{G_{f}^{-1}(0)}: W^{1, p}\left(G_{f}^{-1}(-\infty, 0), \nu_{\lambda}\right) \rightarrow \mathrm{L}^{q}\left(G_{f}^{-1}(0), w_{\lambda} \rho\right)$ for every $q \in[1, p)$ and $f \in X^{*} \backslash\{0\}$. Furthermore, using a similar argument as in Proposition 3.5, we get $\operatorname{Tr}_{G_{f}^{-1}(0)} \varphi \in \mathrm{L}^{q}\left(G_{f}^{-1}(0), \rho\right)$ for every $q \in[1, p)$ and every $\varphi \in W^{1, p}\left(G_{f}^{-1}(-\infty, 0), \nu\right)$.

7.2. A weight without continuous versions. Let $X=\ell_{2}$ the Banach space of square summable sequences and let $\left(v_{k}\right)_{k \in \mathbb{N}}$ its standard orthonormal basis, i.e. $v_{k}$ is the sequence such that $v_{k}(i)=\delta_{i k}$ for every $i, k \in \mathbb{N}$. Let $\mu$ be a centered non-degenerate Gaussian measure on $\ell_{2}$ with covariance operator $Q: \ell_{2} \rightarrow \ell_{2}$ defined by

$$
Q(x)=\left(\frac{x(i)}{2^{i}}\right) .
$$

Such a measure exists, e.g. by [7, Theorem 2.3.1]. The eigenvectors of $Q$ are the vectors $v_{k}$ with respective eigenvalues $2^{-k}$. We will denote by $\left\{e_{n}:=v_{n} / \sqrt{2^{n}}\right\}$ the basis of the Cameron-Martin space associated with $\mu$.

The weight we want to study is $w_{q}(x)=e^{\|x\|_{q}}$ for fixed $q>1$. The first result we need is the fact that $w_{q}$ (actually $\|\cdot\|_{q}$ ) is defined $\mu$-a.e. and in order to show that we need a modification of Fernique's theorem (see [7, Theorem 2.8.5]). Let's start with a definition:

Definition 7.1. Let $\gamma$ be a Gaussian measure on a separable Banach space $X$ and $p \in(0,1]$. A function $g$ measurable with respect to $\operatorname{Borel}(X)$ (the Borel $\sigma$-algebra of $X$ ) is called $\operatorname{Borel}(X)$-measurable $p$ seminorm if there exists a $\operatorname{Borel}(X)$-measurable linear subspace $X_{0} \subseteq X$ of $\gamma$-measure 1 such that $g$ is a $p$-seminorm on $X_{0}$, i.e. $g(x+y) \leq g(x)+g(y)$ for every $x, y \in X_{0}$ and $g(\lambda x)=|\lambda|^{p} g(x)$ for every $x \in X_{0}$ and $\lambda \in \mathbb{R}$.

Our definition differs from the definition of measurable norm of the Cameron-Martin space with the cylindrical Wiener measure in the sense of Gross (see [7, Definition 3.9.2]) since we also consider $p$-seminorm, with $0<p<1$. Observe that our definition of $\operatorname{Borel}(X)$-measurable 1-seminorm agrees with [7, Definition 2.8.1].

The proofs of the following two propositions are similar to the proof of Fernique's theorem. We include the proof of the first one in order to get a self-contained paper.

Proposition 7.2. Let $\mu$ be a centered Gaussian measure on a separable Banach space $X, p \in(0,1]$ and let $g$ be a $\operatorname{Borel}(X)$-measurable p-seminorm. Then

$$
\int_{X} e^{\alpha g(x)^{2}} d \mu(x)<+\infty
$$

for some $\alpha>0$.

Proof. Let $t>\tau>0$. According to [7, Proposition 2.2.10] we have

$$
\begin{gathered}
\mu(\{x \in X \mid g(x) \leq \tau\}) \mu(\{y \in X \mid g(y)>t\})=\int_{\{(x, y) \in X \times X \mid g(x) \leq \tau \text { and } g(y)>t\}} d \mu(x) d \mu(y)= \\
=\int_{\left\{(u, v) \in X \times X \mid g\left(\frac{u-v}{\sqrt{2}}\right) \leq \tau, g\left(\frac{u+v}{\sqrt{2}}\right)>t\right\}} d \mu(u) d \mu(v) \leq \\
\leq \int_{\left\{(u, v) \in X \times X \mid g(u) \geq \frac{t-\tau}{\sqrt{2^{p}}}, g(v)>\frac{t-\tau}{\left.\sqrt{2^{p}}\right\}}\right.} d \mu(u) d \mu(v) .
\end{gathered}
$$

The last inequality follows by $g(u) \geq 2^{-p}(g(u+v)-g(u-v))$ for every $u, v \in X$, indeed

$$
\left\{(u, v) \in X^{2} \mid g\left(\frac{u-v}{\sqrt{2}}\right) \leq \tau, g\left(\frac{u+v}{\sqrt{2}}\right)>t\right\} \subseteq\left\{(u, v) \in X^{2} \mid g(u) \geq \frac{t-\tau}{\sqrt{2^{p}}}, g(v)>\frac{t-\tau}{\sqrt{2^{p}}}\right\} .
$$

Therefore we get

$$
\mu(\{x \in X \mid g(x) \leq \tau\}) \mu(\{y \in X \mid g(y)>t\}) \leq\left(\mu\left\{z \in X \mid g(z)>\frac{t-\tau}{\sqrt{2^{p}}}\right\}\right)^{2}
$$


Since $g<+\infty \mu$-a.e., there exists a positive number $\tau$ such that $c:=\mu(\{x \in X \mid g(x) \leq \tau\})>\frac{1}{2}$. If $c=1$ the statement holds true, indeed $\int_{X} e^{\alpha g(x)^{2}} d \mu(x) \leq e^{\alpha \tau^{2}}$ for every $\alpha>0$. Now assume $c<1$. Let

$$
t_{n}=\tau+\sqrt{2^{p}} t_{n-1} \quad \text { and } \quad t_{0}=\tau .
$$

It is easy to verify that $t_{n}=\tau\left(\sqrt{2^{p}}-1\right)^{-1}\left(2^{p \frac{n+1}{2}}-1\right)$. Letting $p_{n}:=c^{-1} \mu\left(\left\{x \in X \mid g(x)>t_{n}\right\}\right)$, then $p_{n} \leq p_{n-1}^{2}$. By induction we get

$$
\mu\left(\left\{x \in X \mid g(x)>t_{n}\right\}\right) \leq c\left(\frac{1-c}{c}\right)^{2^{n}} .
$$

Let $\alpha>0$. We get

$$
\begin{gathered}
\int_{X} e^{\alpha g(x)^{2}} d \mu(x) \leq \int_{\{x \in X \mid g(x) \leq \tau\}} e^{\alpha g(x)^{2}} d \mu(x)+\sum_{n=0}^{+\infty} e^{\alpha t_{n+1}^{2}} \mu\left(\left\{x \in X \mid t_{n} \leq g(x)<t_{n+1}\right\}\right) \leq \\
\leq c e^{\alpha \tau^{2}}+\sum_{n=0}^{+\infty} e^{\alpha t_{n+1}^{2}} \mu\left(\left\{x \in X \mid g(x) \geq t_{n}\right\}\right) \leq c e^{\alpha \tau^{2}}+\sum_{n=0}^{+\infty} c\left(\frac{1-c}{c}\right)^{2^{n}} \exp \left(\alpha \tau^{2} \frac{\left(2^{p \frac{n+1}{2}}-1\right)^{2}}{\left(\sqrt{2^{p}}-1\right)^{2}}\right)= \\
=c e^{\alpha \tau^{2}}+c \sum_{n=0}^{+\infty} \exp \left(2^{n} \log \left(\frac{1-c}{c}\right)+\alpha \tau^{2}\left(\frac{2^{p \frac{n+1}{2}}-1}{\sqrt{2^{p}}-1}\right)^{2}\right)
\end{gathered}
$$

Observe that by the chain of inequalities $\left(2^{p \frac{n+1}{2}}-1\right)^{2} \leq\left(2^{\frac{n+1}{2}}-1\right)^{2} \leq 2^{n+1}+1$, we get

$$
2^{n} \log \left(\frac{1-c}{c}\right)+\alpha \tau^{2}\left(\frac{2^{p \frac{n+1}{2}}-1}{\sqrt{2^{p}}-1}\right)^{2} \leq 2^{n} \log \left(\frac{1-c}{c}\right)+\alpha \tau^{2} \frac{2^{n+1}+1}{\left(\sqrt{2^{p}}-1\right)^{2}} .
$$

So if we choose $\alpha>0$ such that

$$
\log \left(\frac{1-c}{c}\right)+\frac{2 \alpha \tau^{2}}{\left(\sqrt{2^{p}}-1\right)^{2}}<0
$$

then $\int_{X} e^{\alpha g^{2}} d \mu$ is finite.

Proposition 7.3. Let $\mu$ be a centered Gaussian measure on a locally convex space $X$ and let $g$ be a measurable 1-seminorm. Then $\int_{X} e^{\alpha g(x)} d \mu(x)<+\infty$, for every $\alpha>0$.

Proof. The proof is similar to the proof of Proposition 7.2.

We are now going to consider a notable example.

Proposition 7.4. Let $q>0$ and $\ell_{q}=\left\{\left.x \in \ell_{2}\left|\sum_{i=1}^{+\infty}\right|\left(x, v_{i}\right)_{\ell_{2}}\right|^{q}<+\infty\right\}$ (observe that $\ell_{q}=\ell_{2}$ for every $q \geq 2)$. Then $\mu\left(\ell_{q}\right)=1$ and the function

$$
P_{q}(x):= \begin{cases}\sum_{i=1}^{+\infty}\left|\left(x, v_{i}\right)_{\ell_{2}}\right|^{q} & q \in(0,1) \\ \|x\|_{q} & q \geq 1,\end{cases}
$$

belongs to $\mathrm{L}^{p}\left(\ell_{2}, \mu\right)$, for every $p \geq 1$.

Proof. By [7, Exercise A.3.34], for every $q>0$ the linear space $\ell_{q}$ is measurable with respect to the Borel $\sigma$-algebra in $\ell_{2}$. We have

$$
\int_{\ell_{2}} \sum_{i=1}^{n}\left|\left(x, v_{i}\right)_{\ell_{2}}\right|^{q} d \mu(x)=\sum_{i=1}^{n} \int_{\ell_{2}}\left|\left(x, v_{i}\right)_{\ell_{2}}\right|^{q} d \mu(x) .
$$

Using the change of variable formula (see [7, Equation (A.3.1)]) we get

$$
\int_{\ell_{2}} \sum_{i=1}^{n}\left|\left(x, v_{i}\right)_{\ell_{2}}\right|^{q} d \mu(x)=\sum_{i=1}^{n} \frac{2 \sqrt{2^{i-1}}}{\sqrt{\pi}} \int_{0}^{+\infty} \eta^{q} e^{-2^{i-1} \eta^{2}} d \eta .
$$


Let $c_{q}=2 \sqrt{\pi^{-1}} \int_{0}^{+\infty} t^{q} e^{-t^{2}} d t$. Then

$$
\int_{\ell_{2}} \sum_{i=1}^{n}\left|\left(x, v_{i}\right)_{\ell_{2}}\right|^{q} d \mu(x)=c_{q} \sum_{i=1}^{n}\left(\frac{1}{2^{\frac{q}{2}}}\right)^{i}=c_{q} \frac{2^{\frac{q}{2}(n+1)}-1}{2^{\frac{q}{2} n}\left(2^{\frac{q}{2}}-1\right)} \leq c_{q} \frac{2^{\frac{q}{2}}}{2^{\frac{q}{2}}-1} .
$$

Then by the monotone convergence theorem we get

$$
\int_{\ell_{2}} \sum_{i=1}^{+\infty}\left|\left(x, v_{i}\right)_{\ell_{2}}\right|^{q} d \mu(x)<+\infty
$$

By [7, Theorem 2.5.5] we have $\mu\left(\ell_{q}\right)=1$. Observe that $P_{q}$ is a $\operatorname{Borel}(X)$-measurable $q$-norm, for every $q>0$, then by Proposition 7.2 it belongs to $\mathrm{L}^{p}\left(\ell_{2}, \mu\right)$ for every $p \geq 1$.

Proposition 7.4 implies that $w_{q}=e^{\|x\|_{q}}$ is defined $\mu$-a.e. on $\ell_{2}$ for every $q>1$. Now we need to prove that $w_{q}$ satisfies Hypothesis 1.1. We start with another modification of Fernique's theorem (see [7, Theorem 2.8.5]), which implies that for every $q>1, w_{q} \in \mathrm{L}^{s}\left(\ell_{2}, \mu\right)$ for every $s \geq 1$.

Proposition 7.5. For every $q>1$ consider the function $U_{q}: \ell_{2} \rightarrow \mathbb{R}$ defined by

$$
U(x)= \begin{cases}\|x\|_{q} & x \in \ell_{q} \\ 0 & x \notin \ell_{q}\end{cases}
$$

Then $U \in W^{1, p}\left(\ell_{2}, \mu\right)$ for every $p \geq 1$ and

$$
\partial_{i} U(x)=2^{-\frac{i}{2}} \operatorname{sign}\left(x, v_{i}\right)\left(x, v_{i}\right)^{q-1}\|x\|_{q}^{1-q} \quad \text { p-a.e. }
$$

Proof. For every $n \in \mathbb{N}$ consider the function $\varphi_{n}: \mathbb{R}^{n} \rightarrow \mathbb{R}$ defined as

$$
\varphi_{n}\left(\eta_{1}, \ldots, \eta_{n}\right):=\left(\sum_{i=1}^{n}\left(\eta_{i}^{2}+\frac{1}{2^{n}}\right)^{\frac{q}{2}}\right)^{\frac{1}{q}} .
$$

Let $U_{n}(x):=\varphi_{n}\left(\left(x, v_{1}\right), \ldots,\left(x, v_{n}\right)\right)$ and observe that $U_{n} \rightarrow U$ pointwise $\mu$-a.e. and $U_{n} \in \mathscr{F} \mathscr{C}_{b}^{\infty}\left(\ell_{2}\right)$ for every $n \in \mathbb{N}$. Indeed for every $n \geq 2$

$$
\begin{gathered}
\left(\sum_{i=1}^{n}\left|\left(x, v_{i}\right)\right|^{q}\right)^{\frac{1}{q}} \leq\left|U_{n}(x)\right| \leq\left(\max \left\{1,2^{q-1}\right\} \sum_{i=1}^{n}\left(\left|\left(x, v_{i}\right)\right|^{q}+\frac{1}{2^{\frac{n q}{2}}}\right)\right)^{\frac{1}{q}}= \\
=\max \left\{1,2^{\frac{q-1}{q}}\right\}\left(\frac{n}{2^{\frac{n q}{2}}}+\sum_{i=1}^{n}\left|\left(x, v_{i}\right)\right|^{q}\right)^{\frac{1}{q}} \leq \max \left\{1,2^{\frac{q-1}{q}}\right\}\left(\frac{n}{2^{\frac{n}{2}}}+\left(\sum_{i=1}^{n}\left|\left(x, v_{i}\right)\right|^{q}\right)^{\frac{1}{q}}\right) \\
\leq \max \left\{1,2^{\frac{q-1}{q}}\right\}\left(1+\|x\|_{q}\right) .
\end{gathered}
$$

By Lebesgue's dominated convergence theorem and Proposition 7.4 we get $U_{n} \rightarrow U$ in $\mathrm{L}^{p}\left(\ell_{2}, \mu\right)$ for every $p \geq 1$. Observe that

$$
\partial_{i} U_{n}(x)=\frac{\left(x, v_{i}\right)}{2^{\frac{i}{2}}}\left(\left(x, v_{i}\right)^{2}+\frac{1}{2^{n}}\right)^{\frac{q}{2}-1}\left(\sum_{k=1}^{n}\left(\left(x, v_{k}\right)^{2}+\frac{1}{2^{n}}\right)^{\frac{q}{2}}\right)^{\frac{1}{q}-1},
$$

and $\partial_{i} U_{n}(x) \rightarrow 2^{-\frac{i}{2}} \operatorname{sign}\left(x, v_{i}\right)\left(x, v_{i}\right)^{q-1}\|x\|_{q}^{1-q}$ pointwise $\mu$-a.e. For $1<q \leq 2$

$$
\begin{gathered}
\left|\nabla_{H} U_{n}(x)\right|_{H}=\left(\sum_{i=1}^{+\infty} \frac{\left(x, v_{i}\right)^{2}}{2^{i}}\left(\left(x, v_{i}\right)^{2}+\frac{1}{2^{n}}\right)^{q-2}\left(\sum_{k=1}^{n}\left(\left(x, v_{k}\right)^{2}+\frac{1}{2^{n}}\right)^{\frac{q}{2}}\right)^{\frac{2}{q}-2}\right)^{\frac{1}{2}} \leq \\
\leq 2^{\frac{q-1}{2}}\left(\sum_{i=1}^{+\infty} \frac{\left(x, v_{i}\right)^{2}}{2^{i}}\left(\left(x, v_{i}\right)^{2}+\frac{1}{2^{n}}\right)^{q-2}\right)^{\frac{1}{2}} \leq 2^{\frac{q-1}{2}}\left(\sum_{i=1}^{+\infty} \frac{\left(x, v_{i}\right)^{2 q-2}}{2^{i}}\right)^{\frac{1}{2}} \leq 2^{\frac{q-1}{2}}\left(\sum_{i=1}^{+\infty}\left(x, v_{i}\right)^{q-1}\right) .
\end{gathered}
$$


By Proposition 7.4 the last function is integrable for every $p \geq 1$. If $p>2$ then

$$
\begin{aligned}
\left|\nabla_{H} U_{n}(x)\right|_{H}= & \left(\sum_{i=1}^{+\infty} \frac{\left(x, v_{i}\right)^{2}}{2^{i}}\left(\left(x, v_{i}\right)^{2}+\frac{1}{2^{n}}\right)^{q-2}\left(\sum_{k=1}^{n}\left(\left(x, v_{k}\right)^{2}+\frac{1}{2^{n}}\right)^{\frac{q}{2}}\right)^{\frac{2}{q}-2}\right)^{\frac{1}{2}} \leq \\
& \leq 2^{\frac{q-1}{2}}\left(\sum_{i=1}^{+\infty} \frac{\left(x, v_{i}\right)^{2}}{2^{i}}\left(\left(x, v_{i}\right)^{2}+\frac{1}{2^{n}}\right)^{q-2}\right)^{\frac{1}{2}} \leq \\
\leq & 2^{\frac{q-1}{2}} \max \left\{1,2^{\frac{q-3}{2}}\right\}\left(\sum_{i=1}^{+\infty}\left(\left(x, v_{i}\right)^{2 q-2}+\frac{\left(x, v_{i}\right)^{4}}{2^{n(q-2)}}\right)\right)^{\frac{1}{2}} \leq \\
& \leq 2^{\frac{q-1}{2}} \max \left\{1,2^{\frac{q-3}{2}}\right\}\left(\sum_{i=1}^{+\infty}\left(x, v_{i}\right)^{q-1}+\sum_{i=1}^{+\infty}\left(x, v_{i}\right)^{2}\right) .
\end{aligned}
$$

By Proposition 7.4 the last term belongs to $\mathrm{L}^{p}\left(\ell_{2}, \mu\right)$ for every $p \geq 1$. By the Lebesgue dominated convergence theorem we get $U \in W^{1, p}\left(\ell_{2}, \mu\right)$ for every $p \geq 1$ and $\partial_{i} U(x)=2^{-\frac{i}{2}} \operatorname{sign}\left(x, v_{i}\right)\left(x, v_{i}\right)^{q-1}\|x\|_{q}^{1-q}$ $\mu$-a.e. for every $i \in \mathbb{N}$.

By Proposition 3.6 (applied with the functions $\theta_{n}(\eta)=n \arctan \left(n^{-1} e^{\eta}\right)$, for every $\eta \in \mathbb{R}$ and $n \in \mathbb{N}$, and then using the Lebesgue dominated convergence theorem) we get

$$
\begin{gathered}
\partial_{i} w_{q}(x)=2^{-\frac{i}{2}} \operatorname{sign}\left(x, v_{i}\right)\left(x, v_{i}\right)^{q-1}\|x\|_{q}^{1-q} e^{\|x\|_{q}} \\
\partial_{i} \log w_{q}(x)=2^{-\frac{i}{2}} \operatorname{sign}\left(x, v_{i}\right)\left(x, v_{i}\right)^{q-1}\|x\|_{q}^{1-q} .
\end{gathered}
$$

Proposition 7.3 and Proposition 7.5 yield that the function $w_{q}$ satisfies Hypothesis 1.1 for every $s, t>1$. This implies that it is possible to define $W^{1, p}\left(\ell_{2}, \nu_{q}\right)$ for every $p>1$ (see Definition 3.3). Observe that $\|\cdot\|_{q}$ is not $\mu$-a.e. continuous on $\ell_{2}$ if $q \in(1,2)$.

Using the same argument already used in Example 7.1 it is possible to prove that $S$ satisfies Hypothesis 1.2. The trace operator $\operatorname{Tr}_{S^{-1}(0)}$ maps $W^{1, p}\left(S^{-1}(-\infty, 0), \nu_{q}\right)$ into $\mathrm{L}^{r}\left(S^{-1}(0), w_{q} \rho\right)$ for every $r \in[1, p)$. We do not know whether its range is contained in $\mathrm{L}^{p}\left(S^{-1}(0), w_{q} \rho\right)$. The same considerations are true for the trace operator $\operatorname{Tr}_{G_{f}^{-1}(0)}$ for every $f \in \ell_{2} \backslash\{0\}$.

7.3. An example in $\mathscr{C}[0,1]$. Recall that a function $f: X \rightarrow \mathbb{R}$ from a Banach space $X$ to $\mathbb{R}$ is Gâteaux differentiable in $x \in X$ if for every $y \in X$ the limit

$$
\lim _{t \rightarrow 0} \frac{f(x+t y)-f(x)}{t}
$$

exists and defines a linear (in $y)$ map $((D f) x)(\cdot)$ which is continuous from $X$ to $\mathbb{R}$.

We will use the following result of Aronszajn (see [5, Theorem 1 of Chapter 2] and [19, Theorem $6]$ ).

Theorem 7.6. Suppose that $X$ is a separable real Banach space. If $f: X \rightarrow \mathbb{R}$ is a continuous convex function, then $f$ is Gâteaux differentiable outside a Gaussian null set, i.e. a Borel set $A \subseteq X$ such that $\mu(A)=0$ for every nondegenerate Gaussian measure $\mu$ on $X$.

Consider the classical Wiener measure $P^{W}$ on $\mathscr{C}[0,1]$ (see [7, Example 2.3.11 and Remark 2.3.13] for its construction). Recall that the Cameron-Martin space is the space of the continuous functions $f$ on $[0,1]$ such that $f$ is absolutely continuous, $f^{\prime} \in \mathrm{L}^{2}[0,1]$ and $f(0)=0$. In addition $|f|_{H}=\left\|f^{\prime}\right\|_{\mathrm{L}^{2}[0,1]}$ (see [7, Lemma 2.3.14]). An orthonormal basis of $H$ is given by the functions

$$
f_{n}(\xi)=\sqrt{2 \lambda_{n}} \sin \frac{\xi}{\sqrt{\lambda_{n}}} \quad \text { where } \lambda_{n}=\frac{4}{\pi^{2}(2 n-1)^{2}} \text { for every } n \in \mathbb{N} \text {. }
$$

Consider the weight

$$
w(f)=e^{\|f\|_{\infty}} \quad \text { for every } f \in \mathscr{C}[0,1]
$$


According to [7, Theorem 5.11.2] $w$ is differentiable along $H$. In particular letting

$$
M=\left\{f \in \mathscr{C}[0,1] \mid \text { there exists an unique } \xi \in[0,1] \text { such that }\|f\|_{\infty}=|f(\xi)|\right\},
$$

by Theorem 7.6 and [14, Example 1.6.b] we get that $P^{W}(M)=1$. Furthermore by [6]

$$
\left(\left(D\|\cdot\|_{\infty}\right) f\right)(g)=\operatorname{sign}\left(f\left(\xi_{f}\right)\right) g\left(\xi_{f}\right),
$$

for every $f \in M$ and $g, g_{1}, g_{2} \in \mathscr{C}[0,1]$, where $\xi_{f} \in[0,1]$ is the only point of maximum of the function $|f(\cdot)|$. By [7, Definition 5.2.3 and Proposition 5.4.6(iii)] and by Proposition 3.6 (applied with the functions $\theta_{n}(\eta)=n \arctan \left(n^{-1} e^{\eta}\right)$, for every $\eta \in \mathbb{R}$ and $n \in \mathbb{N}$, and then using the Lebesgue dominated convergence theorem) it can be seen that

$$
\begin{gathered}
\left(\partial_{i} w(f)\right)(\xi)=e^{\|f\|_{\infty} \operatorname{sign}\left(f\left(\xi_{f}\right)\right) f_{i}\left(\xi_{f}\right) ;} \\
\left(\partial_{i} \log w(f)\right)(\xi)=\operatorname{sign}\left(f\left(\xi_{f}\right)\right) f_{i}\left(\xi_{f}\right) .
\end{gathered}
$$

We also have

$$
\begin{aligned}
\|w\|_{L^{s}\left(\mathscr{C}[0,1], P^{W}\right)} & =\left(\int_{\mathscr{C}[0,1]} e^{\left.s\|f\|_{\infty} d P^{W}(f)\right)^{\frac{1}{s}} ;}\right. \\
\left\|\nabla_{H} w\right\|_{L^{s}\left(\mathscr{C}[0,1], P^{W} ; H\right)} & =\left(\int_{\mathscr{C}[0,1]} e^{\left.s\|f\|_{\infty}\left(\sum_{n=1}^{+\infty} f_{n}^{2}\left(\xi_{f}\right)\right)^{s} d P^{W}(f)\right)^{\frac{1}{s}} ;}\right. \\
\|\log w\|_{L^{t}(\mathscr{C}[0,1], P W)} & =\left(\int_{\mathscr{C}[0,1]}\|f\|_{\infty}^{t} d P^{W}(f)\right)^{\frac{1}{t}} ; \\
\left\|\nabla_{H} \log w\right\|_{L^{t}\left(\mathscr{C}[0,1], P P^{W} ; H\right)} & =\left(\int_{\mathscr{C}[0,1]}\left(\sum_{n=1}^{+\infty} f_{n}^{2}\left(\xi_{f}\right)\right)^{t} d P^{W}(f)\right)^{\frac{1}{t}}
\end{aligned}
$$

By Proposition 7.3, (7.1) and (7.3) are finite for every $s, t>1$. By [7, Theorem 5.11.2], (7.2) and (7.4) are finite for every $s>1$. All these results give that the weight $w$ satisfies Hypothesis 1.1 for every $s, t>1$. This implies that it is possible to define $W^{1, p}(\mathscr{C}[0,1], \nu)$ for every $p>1$ (see Definition 3.3).

If we let

$$
S_{1}(f)=\|f\|_{2}-1
$$

then $S_{1} \in \bigcap_{p>1} W^{2, p}\left(\mathscr{C}[0,1], P^{W}\right)$. Furthermore $\nabla_{H} S_{1}(f)=\sum_{i=1}^{+\infty} \frac{\int_{0}^{1} f(\xi) f_{i}(\xi) d \xi}{\|f\|_{2}} f_{i}$ and

$$
\int_{\mathscr{C}[0,1]} \frac{1}{\left|\nabla_{H} S_{1}(f)\right|_{H}^{p}} d P^{W}(f)=\int_{\mathscr{C}[0,1]} \frac{\|f\|_{2}^{p}}{\left(\sum_{j=1}^{+\infty}\left(\int_{0}^{1} f(\xi) f_{n}(\xi) d \xi\right)^{2}\right)^{\frac{p}{2}}} d P^{W}(f) .
$$

Using the Hölder inequality with some $\alpha>1$ we get

$$
\int_{\mathscr{C}[0,1]} \frac{1}{\left|\nabla_{H} S_{1}(f)\right|_{H}^{p}} d P^{W}(f) \leq\left(\int_{\mathscr{C}[0,1]}\|f\|_{2}^{p \alpha^{\prime}} d P^{W}(f)\right)^{\frac{1}{\alpha^{\prime}}}\left(\int_{\mathscr{C}[0,1]} \frac{d P^{W}(f)}{\left(\sum_{j=1}^{+\infty}\left(\int_{0}^{1} f(\xi) f_{n}(\xi) d \xi\right)^{2}\right)^{\frac{p \alpha}{2}}}\right)^{\frac{1}{\alpha}} .
$$

Finally $\int_{\mathscr{C}[0,1]}\|f\|_{2}^{p \alpha^{\prime}} d P^{W}(f)$ is finite by Fernique's theorem (see [7, Theorem 2.8.5]), and recalling that $\mathrm{L}^{2}$-continuous linear functional are also $\mathscr{C}[0,1]$-continuous we can use the change of variable formula (see [7, Equation (A.3.1)]) and obtain

$$
\begin{gathered}
\int_{\mathscr{C}[0,1]} \frac{d P^{W}(f)}{\left(\sum_{j=1}^{+\infty}\left(\int_{0}^{1} f(\xi) f_{n}(\xi) d \xi\right)^{2}\right)^{\frac{p \alpha}{2}}} \leq \int_{\mathscr{C}[0,1]} \frac{d P^{W}(f)}{\left(\sum_{j=1}^{N}\left(\int_{0}^{1} f(\xi) f_{n}(\xi) d \xi\right)^{2}\right)^{\frac{p \alpha}{2}}}= \\
=\frac{1}{\Gamma\left(\frac{p \alpha}{2}\right)} \int_{0}^{+\infty} \xi^{\frac{p \alpha}{2}}-1 \prod_{i=1}^{N} \frac{d \xi}{\sqrt{1+2 \xi \lambda_{i}^{2}}} .
\end{gathered}
$$


S. FERRARI

For $N \in \mathbb{N}$ big enough the last integral is finite and we get that (7.5) is finite for every $p>1$. Thus $S_{1}$ satisfies Hypothesis 1.2. By Corollary 6.3, the trace operator $\operatorname{Tr}_{S^{-1}(0)}$ maps the space $W^{1, p}\left(S_{1}^{-1}(-\infty, 0), \nu\right)$ into $\mathrm{L}^{q}\left(S_{1}^{-1}(0), w \rho\right)$ continuously, for every $q \in[1, p)$.

Fix a finite signed Borel measure $\lambda$ on $[0,1]$ and consider the continuous linear functional

$$
G_{\lambda}(f)=\int_{0}^{1} f(x) d \lambda(x) \quad \text { for every } f \in \mathscr{C}[0,1] .
$$

Using a similar argument as in Proposition 3.5, we get $\operatorname{Tr}_{G_{\lambda}^{-1}(0)} \varphi \in \mathrm{L}^{q}\left(G_{\lambda}^{-1}(0), \rho\right)$ for every $q \in[1, p)$ and every $\varphi \in W^{1, p}\left(G_{\lambda}^{-1}(-\infty, 0), \nu\right)$.

\section{REFERENCES}

[1] D. Addona, G. Cappa, and S. Ferrari. On the domain of elliptic operators defined in subsets of Wiener spaces. ArXiv e-prints, 2017. Submitted. https://arxiv.org/abs/1706.05260.

[2] H. Airault and P. Malliavin. Intégration géométrique sur l'espace de wiener. Bull. Sci. Math., 112:3-52, 1988.

[3] L. Ambrosio, M. Miranda, Jr., and D. Pallara. Sets with finite perimeter in Wiener spaces, perimeter measure and boundary rectifiability. Discrete Contin. Dyn. Syst., 28(2):591-606, 2010.

[4] L. Ambrosio and P. Tilli. Topics on analysis in metric spaces, volume 25 of Oxford Lecture Series in Mathematics and its Applications. Oxford University Press, Oxford, 2004.

[5] N. Aronszajn. Differentiability of Lipschitzian mappings between Banach spaces. Studia Math., 57(2):147-190, 1976.

[6] S. Banach. Theory of linear operations, volume 38 of North-Holland Mathematical Library. North-Holland Publishing Co., Amsterdam, 1987. Translated from the French by F. Jellett, With comments by A. Pełczyński and Cz. Bessaga.

[7] V. I. Bogachev. Gaussian measures, volume 62 of Mathematical Surveys and Monographs. American Mathematical Society, Providence, RI, 1998.

[8] G. Cappa and S. Ferrari. Maximal Sobolev regularity for solutions of elliptic equations in infinite dimensional Banach spaces endowed with a weighted Gaussian measure. J. Differential Equations, 261(12):7099-7131, 2016.

[9] G. Cappa and S. Ferrari. Maximal Sobolev regularity for solutions of elliptic equations in Banach spaces endowed with a weighted Gaussian measure: The convex subset case. J. Math. Anal. Appl., (2017). http://dx.doi.org/10.1016/j.jmaa.2017.09.015.

[10] P. Celada and A. Lunardi. Traces of Sobolev functions on regular surfaces in infinite dimensions. J. Funct. Anal., 266(4):1948-1987, 2014.

[11] G. Da Prato. Kolmogorov equations for stochastic PDEs. Advanced Courses in Mathematics. CRM Barcelona. Birkhäuser Verlag, Basel, 2004.

[12] G. Da Prato and A. Lunardi. Sobolev regularity for a class of second order elliptic PDE's in infinite dimension. Ann. Probab., 42(5):2113-2160, 2014.

[13] G. Da Prato and J. Zabczyk. Second order partial differential equations in Hilbert spaces, volume 293 of London Mathematical Society Lecture Note Series. Cambridge University Press, Cambridge, 2002.

[14] R. Deville, G. Godefroy, and V. Zizler. Smoothness and renormings in Banach spaces, volume 64 of Pitman Monographs and Surveys in Pure and Applied Mathematics. Longman Scientific \& Technical, Harlow; copublished in the United States with John Wiley \& Sons, Inc., New York, 1993.

[15] J. Diestel and J. J. Uhl, Jr. Vector measures. American Mathematical Society, Providence, R.I., 1977. With a foreword by B. J. Pettis, Mathematical Surveys, No. 15.

[16] D. Feyel. Hausdorff-Gauss measures. In Stochastic analysis and related topics, VII (Kusadasi, 1998), volume 48 of Progr. Probab., pages 59-76. Birkhäuser Boston, Boston, MA, 2001.

[17] D. Feyel and A. de La Pradelle. Capacités gaussiennes. Ann. Inst. Fourier (Grenoble), 41(1):49-76, 1991.

[18] E. J. McShane. Extension of range of functions. Bull. Amer. Math. Soc., 40(12):837-842, 1934.

[19] R. R. Phelps. Gaussian null sets and differentiability of Lipschitz map on Banach spaces. Pacific J. Math., 77(2):523531, 1978.

[20] L. Zambotti. Random obstacle problems, volume 2181 of Lecture Notes in Mathematics. Springer, Cham, 2017. Lecture notes from the 45th Probability Summer School held in Saint-Flour, 2015.

(S. Ferrari) Dipartimento di Matematica e Fisica "Ennio de Giorgi", Università del Salento, Via per Arnesano SnC, 73100 Lecce, Italy.

E-mail address: simone.ferrari@unisalento.it 